\title{
Linear precoding via conic optimization for fixed MIMO receivers
}

\author{
Ami Wiesel, Yonina C. Eldar, and Shlomo Shamai (Shitz) \\ Department of Electrical Engineering \\ Technion - Israel Institute of Technology *
}

June 28, 2004

\begin{abstract}
We consider the problem of designing linear precoders for fixed multiple input multiple output (MIMO) receivers. Two different design criteria are considered. In the first, we minimize the transmitted power subject to signal to interference plus noise ratio (SINR) constraints. In the second, we maximize the worst case SINR subject to a power constraint. We show that both problems can be solved using standard conic optimization packages. In addition, we develop conditions for the optimal precoder for both of these problems, and propose two simple fixed point iterations to find the solutions which satisfy these conditions. The relation to the well known downlink uplink duality in the context of joint downlink beamforming and power control is also explored. Our precoder design is general, and as a special case it solves the beamforming problem. In contrast to most of the existing precoders, it is not limited to full rank systems. Simulation results in a multiuser system show that the resulting precoders can significantly outperform existing linear precoders.
\end{abstract}

\section{INTRODUCTION}

Multiple input multiple output (MIMO) systems arise in many modern communication channels, such as multiple user communication [3], and/or multiple antennas channels [4]. It is well known that the use of multiple antennas promises substantial capacity gains when compared to traditional single antenna systems. In order to exploit these gains, the system must deal with the distortion caused by the channel and/or the interference. The conventional way to deal with these distortions is receiver optimization. Recently, the quest for better performance with lower complexity led researchers to also optimize the transmitter [5-10],

\footnotetext{
*e-mails: amiw@tx.technion.ac.il, yonina@ee.technion.ac.il and sshlomo@ee.technion.ac.il. Presented in part in $[1,2]$. The work was supported in part by the NewCom project.
} 
and even to jointly optimize the transmitter and receiver [11-17]. This, as well as new results and algorithms in convex optimization theory [18], have significantly improved state of the art communication systems.

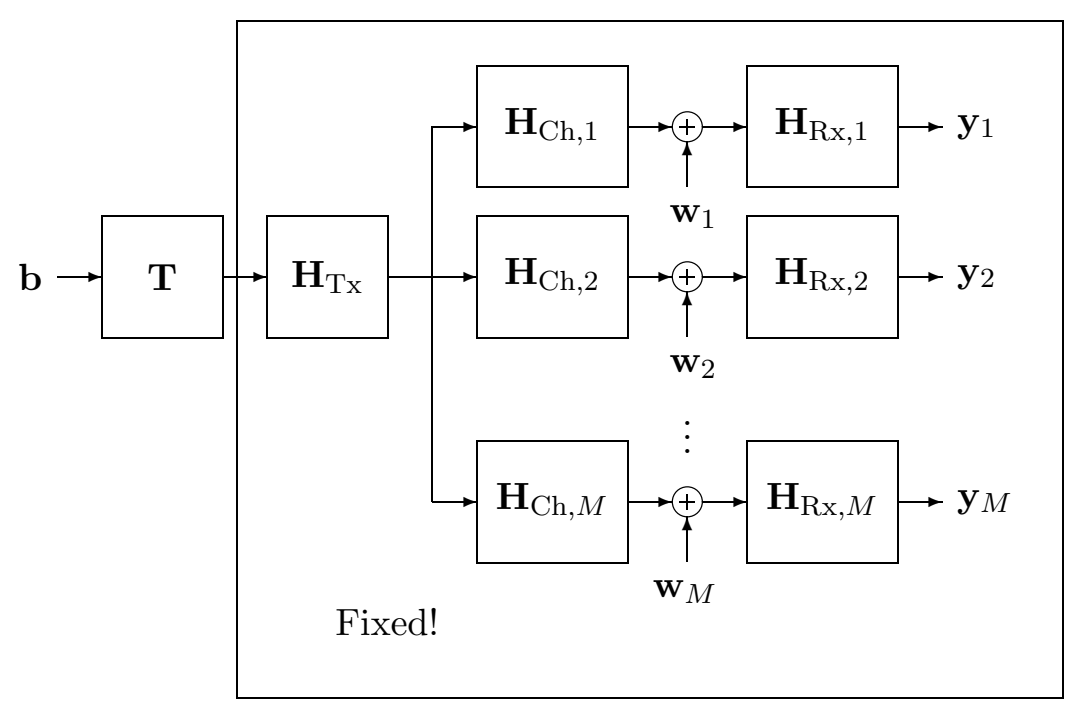

Figure 1: Block diagram of a precoder for a fixed MIMO receiver.

In this paper, we explore the design of a centralized precoder given fixed linear MIMO transmitter, channel and receiver. We define a precoder as a linear transformation on the transmitted symbols. If the precoded symbols are sent as is to the channel, then the precoder is the transmitter itself. However, in general, the precoded symbols may be transformed again before the channel. We refer to this transformation as the transmitter, and we assume that it is a fixed design parameter. An example of a fixed transmitter is the multiuser spreading transmitter, which spreads the symbols using standardized signatures which cannot be altered. The output of the transmitter is then sent over a fixed MIMO channel (or channels), and is received using fixed linear receivers (See Fig. 1). Naturally, a system which jointly optimizes the precoder and receivers will outperform a system with fixed receivers. However, this problem is interesting as it allows improving the system without modifying the receivers. Since optimization techniques are usually costly in terms of complexity, by optimizing only the precoder, we allow for simple low complexity receivers. An appealing application is the downlink channel of a cellular system. The base station, which is less restricted in complexity terms, is optimized and allows better performance without changing the low complexity mobile hand sets. For example, one of our interesting results is that in a symmetric channels, using an optimized precoder achieves the performance of minimum mean squared error (MMSE) receivers while using simple matched filter (MF) receivers.

In our development, we restrict ourselves to design when there is perfect knowledge of the channel 
state information (CSI) at the transmitter. In scenarios when there is no CSI at the transmitter, the common approach is to resort to space time coding or spatial multiplexing. However, in many situations the transmitter has this knowledge. In time division duplex (TDD) it can be estimated, whereas in frequency division duplex (FDD) it can be fedback from the receiver. Therefore, following prior work, we assume that the CSI is perfect and errorless.

One of the first results on optimizing a precoder for a fixed MIMO linear model is due to [5] in the context of multiuser transmission. In that work, two precoders were derived. The precoders applied a linear transformation on the transmitted symbols prior to the spreading. The first precoder was designed to minimize the mean squared error (MSE) between the received vector and the symbols vector. It was then scaled to fit an average power constraint on the output of the transmitter. The second was designed to minimize the MSE subject to an average power constraint. It was observed that the first precoder decorrelates the channel at the transmitter side, and asymptotically outperforms the second. Therefore, it is sometimes referred to as the transmit zero forcing $(\mathrm{ZF})$ precoder.

One of the main drawbacks of the transmit ZF precoder is its degraded performance in low signal to noise ratio (SNR). This phenomena is well known in MIMO reception and multiuser detection [3]. Inverting the channel decreases the output SNR. Therefore, in low SNR, the preferable receiver is the conventional MF which maximizes the SNR. This motivated the design of transmit MF precoders [7] and transmit rakes [19-21]. Clearly, just as in the receiver case, in high SNR, the interference dominates the noise, and the performance of these precoders degrades. Recently, transmit scaled MMSE precoders were derived in [7-10], and tried to compensate for the performance in the different SNR regions. It is important to note that the name transmit MMSE or Transmit Wiener is borrowed from the terminology of receiver design. These receivers do not minimize the MSE. On the contrary, as we explained, the transmit ZF minimizes it [5]. This stems from the fact that the choice of the precoder does not effect the noise since it is placed in the transmitter side.

Other precoders using different kinds of optimization criteria were also derived. We only provide a brief non comprehensive review of the general schemes and do not elaborate on all of the precoders. Variants of the previous precoders were discussed in [22-27]. Linear precoders based on an approximate maximum likelihood approach and maximum asymptotic multiuser efficiency with different power constraints were derived in [28]. A linear precoding technique based on a decomposition approach was proposed in [29]. A linear precoder design for non linear maximum likelihood (ML) receivers was discussed in [30]. Moreover, non linear precoders have also been derived. Among them are the non linear Tomlinson Harashima MIMO 
precoder [6-10,31], the well known "Dirty Paper" precoder [32], and the vector perturbation precoder [33]. Another non linear precoder which optimizes the transmitted symbol's vector itself was derived in [34].

The problem of precoder design is highly related to other problems in the literature. As stated, we investigate the design of linear precoders for fixed linear receivers. A related problem is the problem of jointly optimizing the precoder/transmitter and the receiver, which has been treated, e.g., in $[9,11-17,35,36]$. The design of the optimal signatures (which can be considered as a linear precoding scheme) for matched MMSE receivers was discussed in $[37,38]$, whereas signature design for matched decision feedback receivers was explored in [39]. One of the interesting properties of these joint designs is that maximizing SINR is related to minimizing MSE [15]. Thus, although different criteria have been explored, most of the research was dedicated to variants of the MMSE criterion.

Another related problem is the rank one transmit beamforming design and optimal power control, [40-42]. This problem is well investigated in the literature. The main difference between precoding and transmit beamforming is that in beamforming it is assumed there is no transmitter, i.e., the precoder itself is the transmitter. This simplifies the transmitted power constraints. At first glance it seems that the precoding problem can be solved by addressing the transmit beamforming problem and then compensating for the fixed transmitter. Unfortunately, this is not possible when the transmitter is rank deficient and cannot be inverted. In this aspect ${ }^{1}$, our problem is more general. Unlike the previous references regarding precoding which usually dealt with the MSE criterion and its variants, the beamforming community has successfully managed to optimize SINR based criteria, which are more related to practical performance measures, such as bit error rate (BER) and capacity. This problem is mathematically more difficult than MSE optimization. It was solved using an interesting duality between downlink and uplink beamforming [43-45]. The uplink beamforming problem has been solved before in $[46,47]$. Using the duality, the downlink SINR problem can be handled as well $[41,48,49]$. Recently, a non linear Tomlinson Harashima version of these papers was presented in [50].

In this paper, we integrate the ideas above in the context of MIMO precoding for fixed receivers. As explained before, the design of most of the previous precoders is based on minimizing variants of the common MSE criterion. This criterion is usually computationally attractive and performs quite well. However, as far as the applications are concerned, the interesting and relevant criteria are BER and capacity. It is well known that these are intimately associated with maximizing SINR [3]. Unlike joint optimization, optimizing the system to minimize MSE does not necessarily maximize SINR when the receiver is fixed. Thus, following

\footnotetext{
${ }^{1}$ On the other hand, most of the above references deal with beamforming for rank $r>1$ and are therefore more general than beamforming.
} 
the transmit beamforming approach, we focus on SINR based criteria, and, in particular, try to optimize the worst SINR. We consider two design strategies. The first maximizes the worst SINR subject to an average power constraint. The second minimizes the required average power subject to a constraint on the worst SINR. We prove that the proposed precoders have the attractive property of equal performance among all the sub channels.

Our precoders design is based on the powerful framework of convex optimization theory [18], which allows efficient numerical solutions using standard optimization packages [51]. A brief review of such programs and their standard forms is provided in Section 3. The first contribution of this paper is the casting of the precoder design problems as standard conic optimization packages. The power optimization can be formulated as a Second Order Cone Program (SOCP) [52], or a semi definite program (SDP) [53] (otherwise known as a linear matrix inequalities (LMI) program). This procedure has already been addressed in [42] in the context of beamforming. We generalize it for the case of precoding. Moreover, we show that the SINR optimization can also by formulated as a standard conic program known as the generalized eigenvalue problem (GEVP) [54].

Another contribution of this paper is the derivation of the optimality conditions for both of the design problems. By analyzing the Karush-Kuhn-Tucker (KKT) conditions for conic programs we present simple conditions for optimality. These provide more insight to the problem. We derive a simple expression for the structure of the optimal precoder as a function of the dual variables. The conditions can be used to verify whether a proposed solution is optimal. For example, using these conditions it is easy to show that the so called scaled MMSE precoder proposed in [7-9] does not necessarily maximize the worst SINR, except in the case of a symmetric channel. Another use for these conditions is as a stopping criteria in previous iterative optimization algorithms.

Probably the most important use of the optimality conditions is in deriving new design algorithms. Using the conditions, we provide a simple fixed point iteration which is guaranteed to converge to the solution of the power optimization. As a special case, this simple iteration can solve the well known beamforming problem. This allows a simple solution to the problem without the need for special optimization packages. A similar fixed point iteration is derived for the SINR optimization problem without a convergence proof. In comparison to the downlink-uplink duality based solutions, which consist of inner and outer iterations and eigenvalues optimizations, our fixed point iterations are considerably more appealing. In addition, following [42], we derive an alternative approach for satisfying the optimality conditions in the power optimization through a dual SDP/LMI program. 
One of the advantages of our proposed algorithms is their robustness to the rank of the effective channels. Most of the previous precoders assume a full rank effective channel. For example, one cannot decorrelate the channel in [5] if the channel is rank deficient, as is the case when the number of users is greater than the spreading factor (or the number of transmit antennas). Our design algorithms, both the conic solutions and the fixed point iterations, are indifferent to the rank of the channel, and are therefore applicable to such scenarios as well. In addition, following $[37,38]$ which addressed this problem in the context of optimal sequences design for MMSE receivers, we provide an upper bound for the maximal feasible SINRs in these cases.

An interesting result of our precoders is their performance in symmetric systems. As already mentioned, in this case, our precoders admits a simple closed form expression that has already been derived in [7-9] through different considerations and for general channels. Using our optimality conditions, it is easy to show that these precoders maximize the worst SINR in symmetric channels. A realistic example of such systems is a code division multiple access (CDMA) scheme, using pseudonoise (PN) sequences as signatures. Moreover, we analytically show that the achievable SINRs using these precoders with Matched Filter (MF) receivers is identical to those obtained by using MMSE receivers with no precoders. This result is interesting, as it allows for each user to use a simple receiver that does not require neither the knowledge of all the other signatures nor a matrix inversion. It is important to note that this feature does not extend to non symmetric channels.

The paper is organized as follows. We begin in Section 2 by introducing the problem formulation. A brief review of conic optimization is provided in Section 3. The power optimization problem is explored in Section 4, in which we discuss its feasibility, and provide standard conic optimization solutions. In order to improve our design algorithms and in order to gain more insight into the problem, we then provide optimality conditions, and suggest a simple fixed point iteration for finding the variables that satisfy them. Next, in Section 5 we follow the same steps while addressing the SINR optimization problem. A few special cases for which a closed form solution exists are explored in Section 6. In Section 7, we illustrate the use of the aforementioned precoders in the context of multiple user communication systems.

The following notation is used. Boldface upper case letters denote matrices, boldface lower case letters denote column vectors, and standard lower case letters denote scalars. The superscripts $(\cdot)^{T},(\cdot)^{H},(\cdot)^{-1}$ and $(\cdot)^{\dagger}$ denote the transpose, the Hermitian, the matrix inverse operators, and the Moore Penrose pseudoinverse, respectively. $[\mathbf{X}]_{i, j}$ denotes the (ith,jth) element of the matrix $\mathbf{X}$. By diag $\left\{x_{i}\right\}$ we denote a diagonal matrix with $x_{i}$ being the $(i$ th, $i$ th $)$ element, by vec $(\mathbf{X})$ we denote stacking the elements of $\mathbf{X}$ in one long column 
vector, by $\mathbf{e}_{i}$ we denote a zeros vector with a one at the $i$ 'th element, by $\mathbf{1}$ we denote an all ones vector, and by $\mathbf{I}$ we denote the identity matrix of appropriate size. $\operatorname{Tr}\{\cdot\},|\cdot|,\|\cdot\|$ and ||$|\cdot|||$ denote the trace operator, the Kronecker product, the absolute value operator, the standard Euclidean norm, and the induced matrix norm, respectively. Finally, $\mathbf{X} \succeq 0$ denotes that the matrix $\mathbf{X}$ is a Hermitian positive semi definite matrix, and $\mathcal{N}\{\cdot\}$ denotes the Null space operator.

\section{PROBLEM FORMULATION}

Consider a general, block oriented, MIMO communication system with a centralized transmitter. At each time instant, a block of symbols is modulated and transmitted over a channel. The possibly distorted output is then processed at the receiver in a linear fashion, as depicted in Fig. 1. Denoting by $\mathbf{y}_{i}$ the length $L$ output of the $i$ 'th receiver, for $i=1, \cdots, M$, we have that:

$$
\left[\begin{array}{c}
\mathbf{y}_{1} \\
\vdots \\
\mathbf{y}_{M}
\end{array}\right]=\left[\begin{array}{c}
\mathbf{H}_{\mathrm{Rx}, 1} \mathbf{H}_{\mathrm{Ch}, 1} \\
\vdots \\
\mathbf{H}_{\mathrm{Rx}, M} \mathbf{H}_{\mathrm{Ch}, M}
\end{array}\right] \mathbf{H}_{\mathrm{Tx}} \mathbf{b}+\left[\begin{array}{c}
\mathbf{H}_{\mathrm{Rx}, 1} \mathbf{w}_{1} \\
\vdots \\
\mathbf{H}_{\mathrm{Rx}, M} \mathbf{w}_{M}
\end{array}\right] \text {, }
$$

where the matrices $\mathbf{H}_{\mathrm{Rx}, i}$ and $\mathbf{H}_{\mathrm{Ch}, i}$ denote the receiver and channel associated with the $i$ 'th user, the matrix $\mathbf{H}_{\mathrm{Tx}}$ is the centralized transmitter, $\mathbf{b}$ is the length $K=M \cdot L$ vector of independent, and unit variance transmitted symbols, and $\mathbf{w}_{i}$ are the noise vectors. This system model is quite general. The noise vectors may be correlated or even identical to each other, and the channels are completely arbitrary. The only restriction is that the transmitter is centralized and has access to all of the $K$ transmit components.

Let us present two specific examples for which the following problem formulation holds:

- CDMA system - Consider a multiple user downlink system using CDMA. At each time period, the base station transmits $K$ symbols using an $N \times K$ signature matrix $\mathbf{H}_{\mathrm{Tx}}=\mathbf{S}$ with columns $\mathbf{s}_{i}$. For simplicity, we assume ideal channels $\mathbf{H}_{\mathrm{Ch}, i}=\mathbf{I}$ and conventional matched filter receivers $\mathbf{H}_{\mathrm{Rx}, i}=\mathbf{s}_{i}^{H}$ where $\mathbf{s}_{i}$ denotes the $i$ th column of $\mathbf{S}$. Denoting by $y_{i}$ the output of the $i$ 'th user's receiver, we have that

$$
\left[\begin{array}{c}
y_{1} \\
\vdots \\
y_{K}
\end{array}\right]=\left[\begin{array}{c}
\mathbf{s}_{1}^{H} \\
\vdots \\
\mathbf{s}_{K}^{H}
\end{array}\right] \mathbf{S b}+\left[\begin{array}{c}
\mathbf{s}_{1}^{H} \mathbf{w}_{1} \\
\vdots \\
\mathbf{s}_{K}^{H} \mathbf{w}_{K}
\end{array}\right]
$$

which is clearly a special case of (1). 
- Beamforming - Consider a multiuser system incorporating multiple antennas. At each time period, $K$ symbols are modulated, without any transformation at the transmitter $\mathbf{H}_{\mathrm{Tx}}=\mathbf{I}$, through $K$ transmit antennas to $K$ users, each using one receive antenna. The different pathes between the transmit antennas and the $i$ 'th user are represented in the length $K$ vectors $\mathbf{H}_{\mathrm{Ch}, i}=\mathbf{h}_{i}^{H}$. Denoting by $y_{i}$ the output of the $i$ 'th user's receiver, we have that

$$
\left[\begin{array}{c}
y_{1} \\
\vdots \\
y_{K}
\end{array}\right]=\left[\begin{array}{c}
\mathbf{h}_{1}^{H} \\
\vdots \\
\mathbf{h}_{K}^{H}
\end{array}\right] \mathbf{b}+\left[\begin{array}{c}
w_{1} \\
\vdots \\
w_{K}
\end{array}\right]
$$

which is again a special case of (1).

In the sequel, we will assume that the transmitter $\mathbf{H}_{\mathrm{Tx}}$, the channels $\mathbf{H}_{\mathrm{Ch}, i}$ and the receivers $\mathbf{H}_{\mathrm{Rx}, i}$ are fixed, and cannot be altered due to budget restrictions, standardization, or physical problems. Given this fixed structure, we will try to improve the performance of such systems by introducing a linear precoder. The precoder, denoted by $\mathbf{T}$, linearly transforms the original symbol vector prior to the transmission, so that the outputs of the receiver are now given by

$$
\left[\begin{array}{c}
\mathbf{y}_{1} \\
\vdots \\
\mathbf{y}_{M}
\end{array}\right]=\left[\begin{array}{c}
\mathbf{H}_{\mathrm{Rx}, 1} \mathbf{H}_{\mathrm{Ch}, 1} \\
\vdots \\
\mathbf{H}_{\mathrm{Rx}, M} \mathbf{H}_{\mathrm{Ch}, M}
\end{array}\right] \mathbf{H}_{\mathrm{Tx}} \mathbf{T b}+\left[\begin{array}{c}
\mathbf{H}_{\mathrm{Rx}, 1} \mathbf{w}_{1} \\
\vdots \\
\mathbf{H}_{\mathrm{Rx}, M} \mathbf{w}_{M}
\end{array}\right]
$$

For ease of representation, we will use the following notation:

$$
\mathbf{y}=\mathbf{H T b}+\mathbf{w}
$$

where $\mathbf{H}=\mathbf{H}_{\mathrm{RxCh}} \mathbf{H}_{\mathrm{Tx}}$ and

$$
\mathbf{y}=\left[\begin{array}{c}
\mathbf{y}_{1} \\
\vdots \\
\mathbf{y}_{M}
\end{array}\right], \quad \mathbf{H}_{\mathrm{RxCh}}=\left[\begin{array}{c}
\mathbf{H}_{\mathrm{Rx}, 1} \mathbf{H}_{\mathrm{Ch}, 1} \\
\vdots \\
\mathbf{H}_{\mathrm{Rx}, M} \mathbf{H}_{\mathrm{Ch}, M}
\end{array}\right], \quad \mathbf{w}=\left[\begin{array}{c}
\mathbf{H}_{\mathrm{Rx}, 1} \mathbf{w}_{1} \\
\vdots \\
\mathbf{H}_{\mathrm{Rx}, M} \mathbf{w}_{M}
\end{array}\right]
$$

Our goal is to improve the system performance by optimally designing the precoder. The system performance is usually quantified by its quality of service (QoS) and the resources it uses. The most common QoS metrics are BER and capacity, both of which are highly related to the output SINRs, and in particular 
to the worst SINR. In our model, the output SINR of the $i$ 'th sub channel is defined as:

$$
\mathrm{SINR}_{i}=\frac{E\left\{\left|[\mathbf{H T}]_{i, i}\right|^{2}\left|b_{i}\right|^{2}\right\}}{E\left\{\left[\left|y_{i}\right|^{2}-\left|[\mathbf{H T}]_{i, i}\right|^{2}\left|b_{i}\right|^{2}\right]\right\}}=\frac{\left|[\mathbf{H T}]_{i, i}\right|^{2}}{\sum_{i \neq j}\left|[\mathbf{H T}]_{i, j}\right|^{2}+\sigma_{i}^{2}}, \quad i=1, \cdots, K,
$$

where $\sigma_{i}^{2}=E\left\{\left|w_{i}\right|^{2}\right\}>0$. Another range of criteria deal with the use of system resources, e.g., peak to average ratio, or maximal transmitted power. The most common resource measure is average transmitted power, which is defined as:

$$
P=E\left\{\left\|\mathbf{H}_{\mathrm{Tx}} \mathbf{T b}\right\|^{2}\right\}=\operatorname{Tr}\left\{\mathbf{T}^{H} \mathbf{H}_{\mathrm{Tx}}^{H} \mathbf{H}_{\mathrm{Tx}} \mathbf{T}\right\}
$$

It is easy to see that the SINRs metrics and average power metric conflict. One cannot maximize the SINRs while also minimizing the power, and vice versa. Depending on the application, the designer must decide which criteria is stricter. We therefore consider one of the following two complementary strategies. The first optimization strategy seeks to minimize the average transmitted power subject to QoS constraints. This criterion is interesting from a system level perspective. Given the required QoS, the system tries to satisfy it with minimum transmitted power [16], [41]:

$$
\mathcal{P}\left(\gamma_{o}\right)= \begin{cases}\min _{\mathbf{T}} & \operatorname{Tr}\left\{\mathbf{T}^{H} \mathbf{H}_{\mathrm{Tx}}^{H} \mathbf{H}_{\mathrm{Tx}} \mathbf{T}\right\} \\ \text { s.t. } & \min _{i} \frac{\left|[\mathbf{H T}]_{i, i}\right|^{2}}{\sum_{i \neq j}\left|[\mathbf{H T}]_{i, j}\right|^{2}+\sigma_{i}^{2}} \geq \gamma_{o}\end{cases}
$$

where $\gamma_{o}>0$ is the given worst SINR constraint.

The second strategy is maximizing the minimal SINR subject to a power constraint [48], [15]. This problem formulation is interesting when the power constraint is a strict system restriction which cannot be relaxed. In this case, the problem can be formulated as

$$
\mathcal{S}\left(P_{o}\right)= \begin{cases}\max _{\mathbf{T}} & \min _{i} \frac{\left|[\mathbf{H T}]_{i, i}\right|^{2}}{\sum_{i \neq j}\left|[\mathbf{H T}]_{i, j}\right|^{2}+\sigma_{i}^{2}} \\ \text { s.t. } & \operatorname{Tr}\left\{\mathbf{T}^{H} \mathbf{H}_{\mathrm{Tx}}^{H} \mathbf{H}_{\mathrm{Tx}} \mathbf{T}\right\} \leq P_{o}\end{cases}
$$

where $P_{o}>0$ is the given power constraint.

Note that although we are optimizing the minimum SINR in both problems, in the sequel, we will show that at the optimal solution of both problems all users will attain equal SINRs. In other words, the above design criteria both promise fairness among all the sub streams. This is an important property in MIMO communication systems. In systems where some streams demand different QoS, e.g., systems with voice 
and data streams, the designer can replace each $\operatorname{SINR}_{i}$ in the optimizations with $\operatorname{SINR}_{i} / \rho_{i}$ where $\rho_{i}$ are constant weights that denote the importance of the sub streams. This will ensure weighted fairness among the streams.

One of the main observations of our work is that both optimization problems (9) and (10) can be solved using standard conic optimization algorithms. Therefore, in the following section, we review these algorithms.

\section{REVIEW OF CONIC OPTIMIZATION}

In recent years, there has been considerable progress and development of efficient algorithms for solving a variety of optimization problems. In order to use these algorithms, one must reformulate the problem into a standard form which the algorithms are capable of dealing with. In this section, we will briefly review the three formulations which we use in the paper: SOCP, SDP and GEVP programming.

The most widely researched field in optimization is convex optimization. A convex program is a program with a convex objective function and convex constraints. It is well known that in such programs a local minimum is also a global minimum. Thus, the global minimum can be found by any "Hill Climbing" or "Gradient Descent" algorithm. The most common convex program is probably the Linear Program (LP) [18], i.e., an optimization with a linear objection function and linear (affine) constraints. Recent advances in convex optimization generalize the results and algorithms of LPs to more complicated convex programs. Special attention is given to conic programs, i.e., LPs with generalized inequalities. The two standard conic programs are SOCP and SDP optimization. The standard form of an SOCP is [52]:

$$
\text { SOCP : } \begin{cases}\min _{\mathbf{x}} & \mathbf{f}^{H} \mathbf{x} \\
\text { s.t. } & {\left[\begin{array}{c}
\mathbf{c}_{i}^{H} \mathbf{x}+d_{i} \\
\mathbf{A}_{i}^{H} \mathbf{x}+\mathbf{b}_{i}
\end{array}\right] \succeq_{K} 0, \quad i=1, \cdots, N,}\end{cases}
$$

where the optimization variable is the vector $\mathbf{x}$ of length $n$ and $\mathbf{f}, \mathbf{A}_{i}, \mathbf{b}_{i}, \mathbf{c}_{i}$ and $d_{i}$ for $i=1, \cdots, N$ are the data parameters of appropriate sizes. The notation $\succeq_{K}$ denotes the following generalized inequality:

$$
\left[\begin{array}{l}
z \\
\mathbf{z}
\end{array}\right] \succeq_{K} 0 \quad \Leftrightarrow \quad\|\mathbf{z}\| \leq z .
$$


The standard form of an SDP is [53]:

$$
\operatorname{SDP}: \begin{cases}\min _{\mathbf{x}} & \mathbf{f}^{H} \mathbf{x} \\ \text { s.t. } & \mathbf{A}(\mathbf{x}) \succeq 0\end{cases}
$$

where $\mathbf{A}(\mathbf{x})=\mathbf{A}_{0}+\sum_{i=1}^{n} x_{i} \mathbf{A}_{i}$ is an Hermitian matrix that depends affinely on $\mathbf{x}$. The data parameters are the Hermitian matrices $\mathbf{A}_{i}$ for $i=0, \cdots, n$. The notation $\succeq$ denotes the positive semi definite generalized inequality. A simple case of an SDP is an SOCP. This stems from the property that any SOC inequality can be written as an LMI [18]:

$$
\left[\begin{array}{c}
\mathbf{c}_{i}^{H} \mathbf{x}+d_{i} \\
\mathbf{A}_{i}^{H} \mathbf{x}+\mathbf{b}_{i}
\end{array}\right] \succeq_{K} 0 \Leftrightarrow\left[\begin{array}{cc}
\mathbf{c}_{1}^{H} \mathbf{x}+d_{1} & \mathbf{x}^{H} \mathbf{A}_{1}+\mathbf{b}_{1}^{H} \\
\mathbf{A}_{1}^{H} \mathbf{x}+\mathbf{b}_{1} & \left(\mathbf{c}_{1}^{H} \mathbf{x}+d_{1}\right) \mathbf{I}
\end{array}\right] \succeq 0 .
$$

A common optimization package designed to solve SOCP and SDP is SEDUMI [51].

Although most of the research in the field of optimization concerns convex programs, due to their importance, some cases of non convex problems have also been investigated. Among them is the generalized eigenvalue minimization program (GEVP) [54], which is not convex but can still be efficiently solved. Its standard form is

$$
\text { GEVP : } \begin{cases}\min _{\beta, \mathbf{x}} & \beta \\ \text { s.t. } & \beta \mathbf{B}(\mathbf{x})-\mathbf{A}(\mathbf{x}) \succeq 0 ; \\ & \mathbf{B}(\mathbf{x}) \succeq 0 ; \\ & \mathbf{C}(\mathbf{x}) \succeq 0,\end{cases}
$$

where $\mathbf{A}(\mathbf{x})=\mathbf{A}_{0}+\sum_{i=1}^{n} x_{i} \mathbf{A}_{i}, \mathbf{B}(\mathbf{x})=\mathbf{B}_{0}+\sum_{i=1}^{n} x_{i} \mathbf{B}_{i}$ and $\mathbf{C}(\mathbf{x})=\mathbf{C}_{0}+\sum_{i=1}^{n} x_{i} \mathbf{C}_{i}$ are Hermitian matrices that depend affinely on $\mathbf{x}$. The data parameters are the Hermitian matrices $\mathbf{A}_{i}, \mathbf{B}_{i}$ and $\mathbf{C}_{i}$ for $i=0, \cdots, n$. The name of the GEVP arises from its resemblance to the well known problem of minimizing the maximal generalized eigenvalue of the pencil $[\mathbf{A}, \mathbf{B}]$, i.e., minimizing the largest $\beta$ such that $\mathbf{A v}=\beta \mathbf{B v}$. It is easy to show that this problem can be expressed as

$$
\begin{cases}\min _{\beta} & \beta \\ \text { s.t. } & \beta \mathbf{B}-\mathbf{A} \succeq 0,\end{cases}
$$

which is of course a simple SDP. The GEVP generalizes this program to the case where $\mathbf{A}$ and $\mathbf{B}$ also depend on the optimization variables. 


\section{POWER OPTIMIZATION}

In this section, we consider the power optimization subject to SINR constraints, i.e., the $\mathcal{P}$ problem of (9). We begin in Section 4.1 by discussing its feasibility, and then provide a few alternative approaches for its solution. In particular, in Section 4.2, we derive a solution to the problem which is based on standard SOCP or SDP optimization packages. Next, in Section 4.3, we develop optimality conditions for this problem, and use them to derive two alternative solutions. For completeness, in Section 4.4, we discuss the downlink uplink duality in the context of the power optimization.

\subsection{FEASIBILITY}

The first important property of any optimization problem is its feasibility (admissibility), i.e., whether a solution exists. In other words, we need to verify whether for a given $\gamma_{o}$ there exists a $\mathbf{T}$ such that

$$
\min _{i} \frac{\left|[\mathbf{H T}]_{i, i}\right|^{2}}{\sum_{i \neq j}\left|[\mathbf{H T}]_{i, j}\right|^{2}+\sigma_{i}^{2}} \geq \gamma_{o}
$$

It is easy to see that the SINRs are bounded by the signal to interference ratios (SIR):

$$
\frac{\left|[\mathbf{H T}]_{i, i}\right|^{2}}{\sum_{i \neq j}\left|[\mathbf{H T}]_{i, j}\right|^{2}+\sigma_{i}^{2}} \leq \frac{\left|[\mathbf{H T}]_{i, i}\right|^{2}}{\sum_{i \neq j}\left|[\mathbf{H T}]_{i, j}\right|^{2}}, \quad i=1, \cdots, K,
$$

with equality if and only if $\sigma_{i}^{2}=0$ for $i=1, \cdots, K$, i.e., in a zero noise environment. By scaling $\mathbf{T}$ to $a \mathbf{T}$ for large enough $a>0$, the difference between the SIRs and the SINRs can be made insignificant. Therefore, for the sake of examining the feasibility, the interesting case is the zero noise environment. A condition for the feasibility in this case is provided in the following lemma.

Lemma 1. There exists a $\mathbf{T}$ such that (17) holds only if

$$
\gamma_{o} \leq \frac{1}{\frac{K}{\operatorname{rank}(\mathbf{H})}-1}
$$

Proof. In order to prove the lemma we must upper bound the minimal SIR:

$$
\min _{i} \frac{\left|[\mathbf{H T}]_{i, i}\right|^{2}}{\sum_{i \neq j}\left|[\mathbf{H T}]_{i, j}\right|^{2}}=\min _{i} \frac{1}{\frac{1}{\xi_{i}}-1}=\frac{1}{\frac{1}{\min _{i} \xi_{i}}-1}
$$


where $\xi_{i}=\frac{\left|[\mathbf{H T}]_{i, i}\right|^{2}}{\left[\mathbf{H T T}^{H} \mathbf{H}^{H}\right]_{i, i}}$, and we have used the monotonicity of $f(a)=\frac{1}{\frac{1}{a}-1}$ in $a \leq 1$. Due to monotonicity, we can bound $f(a)$ by bounding its argument. Thus, we now develop a bound on the minimum $\xi_{i}$. Let HT have a singular values decomposition $(\mathrm{SVD}) \mathbf{H T}=\mathbf{U} \boldsymbol{\Lambda} \mathbf{V}^{H}$, where $\mathbf{U}$ and $\mathbf{V}$ are unitary $K \times r$ matrices, $\boldsymbol{\Lambda}$ is an $r \times r$ diagonal matrix, and $r=\operatorname{rank}(\mathbf{H T})$. Then,

$$
\xi_{i}=\frac{\left|\mathbf{u}_{i}^{H} \boldsymbol{\Lambda} \mathbf{v}_{i}\right|^{2}}{\mathbf{u}_{i}^{H} \boldsymbol{\Lambda}^{2} \mathbf{u}_{i}}, \quad i=1, \cdots, K
$$

where $\mathbf{u}_{i}$ and $\mathbf{v}_{i}$ are the $i$ 'th columns of $\mathbf{U}^{H}$ and $\mathbf{V}^{H}$, respectively. For every $i=1, \cdots, K$, we can bound (21) by applying the Cauchy-Schwarz inequality to the vectors $\boldsymbol{\Lambda} \mathbf{u}_{i}$ and $\mathbf{v}_{i}$

$$
\left|\mathbf{u}_{i}^{H} \boldsymbol{\Lambda} \mathbf{v}_{i}\right|^{2} \leq\left(\mathbf{v}_{i}^{H} \mathbf{v}_{i}\right)\left(\mathbf{u}_{i}^{H} \boldsymbol{\Lambda}^{2} \mathbf{u}_{i}\right), \quad i=1, \cdots, K
$$

Since $\mathbf{v}_{i}^{H} \mathbf{v}_{i}=\left[(\mathbf{H T})^{\dagger} \mathbf{H T}\right]_{i, i}$, we conclude that

$$
\xi_{i} \leq\left[(\mathbf{H T})^{\dagger} \mathbf{H T}\right]_{i, i}, \quad i=1, \cdots, K
$$

Thus, the minimum $\xi_{i}$ is bounded by

$$
\min _{i} \xi_{i} \leq \frac{1}{K} \sum_{i=1}^{K} \xi_{i} \leq \frac{1}{K} \sum_{i=1}^{K}\left[(\mathbf{H T})^{\dagger} \mathbf{H T}\right]_{i, i}=\frac{1}{K} \operatorname{Tr}\left\{(\mathbf{H T})^{\dagger} \mathbf{H T}\right\}=\frac{\operatorname{rank}(\mathbf{H T})}{K} \leq \frac{\operatorname{rank}(\mathbf{H})}{K}
$$

Substituting (24) into (20) yields the required condition on $\gamma_{o}$.

If the effective channel $\mathbf{H}$ is full rank, then the lemma results in $\gamma_{o} \leq \infty$, i.e., any SIR is feasible. This is easily verified as the condition in (17) can be satisfied by choosing $\mathbf{T}=a \mathbf{H}^{-1}$ for large enough $a>0$. This choice of precoder inverts the channel and eliminates all interference.

Unfortunately, when the effective channel is rank deficient, the interference cannot be eliminated, and there is an upper bound on the maximal SIRs. A similar lemma was provided in [37] in the context of optimal signature design using MMSE receivers (which is a special case of a MIMO system). There, it was shown that the above condition is necessary and sufficient for feasibility using MMSE receivers. In our case, the receivers are fixed, and therefore the condition is only necessary. In general, we cannot always attain the bound when the receiver is fixed. Two simple examples for channels in which the bound cannot be achieved are a diagonal $\mathbf{H}$ with $K-\operatorname{rank}(\mathbf{H})$ diagonal zeros, or a channel $\mathbf{H}$ with two identical rows. In both of these examples, it is easy to see that, no matter what the precoder is, we will not attain the bound. 
Nonetheless, experimenting with arbitrary channels shows that in almost all practical channels the bound can be achieved even for a fixed sub optimal receiver. For example, consider a rank $K-1$ channel $\mathbf{H}$, with the normalized null vector $\mathbf{u} \in \mathcal{N}\left\{\mathbf{H}^{H}\right\}$. Except for the case in which $u_{i}=0$ for some $i=1, \cdots, K$, the bound can always be attained by choosing

$$
\mathbf{T}=\mathbf{H}^{\dagger} \operatorname{diag}\left\{1 / \mathbf{u}_{i}\right\} \mathbf{Q}
$$

where

$$
[\mathbf{Q}]_{i, j}=\left\{\begin{array}{cc}
1, & i=j \\
-\frac{1}{K-1}, & j \neq i .
\end{array}\right.
$$

This is easily shown by considering the following chain:

$$
\mathbf{H T}=\mathbf{H H}^{\dagger} \operatorname{diag}\left\{1 / \mathbf{u}_{i}\right\} \mathbf{Q}=\operatorname{diag}\left\{1 / \mathbf{u}_{i}\right\} \mathbf{Q},
$$

where we have used $\mathbf{H H}^{\dagger}=\mathbf{I}-\mathbf{u u}^{H}$ and the fact that $\mathbf{1} \in \mathcal{N}\{\mathbf{Q}\}$. Substituting the above $\mathbf{H T}$ into the SIRs yields the maximal SIRs in rank $K-1$ channels:

$$
\frac{\left|[\mathbf{H T}]_{i, i}\right|^{2}}{\sum_{i \neq j}\left|[\mathbf{H T}]_{i, j}\right|^{2}}=\frac{1}{\frac{K}{K-1}-1}, \quad i=1, \cdots, K .
$$

\subsection{CONIC OPTIMIZATION SOLUTION}

We now show that the $\mathcal{P}$ problem of (9) can be represented as a standard conic optimization program. Thus, using off the shelf optimization packages, we can numerically verify its feasibility, and find its optimal solution. In order to use the standard forms of the conic programs, we must cast our problem constraints using the standard notations described in Section 3.

Using a slack variable, the program can be rewritten as

$$
\mathcal{P}\left(\gamma_{o}\right): \begin{cases}\min _{\mathbf{T}, P_{o}} & P_{o} \\ \text { s.t. } & \frac{\left|[\mathbf{H T}]_{i, i}\right|^{2}}{\sum_{i \neq j}\left|[\mathbf{H T}]_{i, j}\right|^{2}+\sigma_{i}^{2}} \geq \gamma_{o}, \quad i=1, \cdots, K \\ & \operatorname{Tr}\left\{\mathbf{T}^{H} \mathbf{H}_{\mathrm{Tx}}^{H} \mathbf{H}_{\mathrm{Tx}} \mathbf{T}\right\} \leq P_{o} .\end{cases}
$$

The argument $\mathbf{T}$ of the $\mathcal{P}$ program is optimal up to a diagonal phase scaling on the right, i.e., if $\mathbf{T}$ is 
optimal, then $\mathbf{T} \operatorname{diag}\left\{e^{j \phi_{i}}\right\}$, where $\phi_{i}$ for $i=1, \cdots, K$ are arbitrary phases, is also optimal. This is easy to verify, as the phases do not change the objective nor the constraints. Therefore, we can restrict ourselves to precoders in which $[\mathbf{H T}]_{i, i} \geq 0$ for $i=1, \cdots, K$, i.e., each has a non negative real part, and a zero imaginary part. Taking this into account, we now recast the SINR constraints in standard form. Rearranging the constraints and using matrix notations, the constraints yield

$$
\left(1+\frac{1}{\gamma_{o}}\right)\left|[\mathbf{H T}]_{i, i}\right|^{2} \geq\left[\mathbf{H} \mathbf{T} \mathbf{T}^{H} \mathbf{H}^{H}\right]_{i, i}+\sigma_{i}^{2}, \quad i=1, \cdots, K,
$$

or

$$
\left(1+\frac{1}{\gamma_{o}}\right)\left|[\mathbf{H T}]_{i, i}\right|^{2} \geq\left\|\begin{array}{c}
\mathbf{T}^{H} \mathbf{H}^{H} \mathbf{e}_{i} \\
\sigma_{i}
\end{array}\right\|^{2}, \quad i=1, \cdots, K .
$$

Since $[\mathbf{H T}]_{i, i} \geq 0$ for $i=1, \cdots, K$, we can take the square root of $\left|[\mathbf{H T}]_{i, i}\right|^{2}$ resulting in

$$
\sqrt{1+\frac{1}{\gamma_{o}}}[\mathbf{H T}]_{i, i} \geq\left\|\begin{array}{c}
\mathbf{T}^{H} \mathbf{H}^{H} \mathbf{e}_{i} \\
\sigma_{i}
\end{array}\right\|, \quad i=1, \cdots, K,
$$

which can be written as the SOCs

$$
\left[\begin{array}{c}
\sqrt{1+\frac{1}{\gamma_{o}}}[\mathbf{H T}]_{i, i} \\
\mathbf{T}^{H} \mathbf{H}^{H} \mathbf{e}_{i} \\
\sigma_{i}
\end{array}\right] \succeq_{K} 0, \quad i=1, \cdots, K .
$$

The power constraint

$$
\operatorname{Tr}\left\{\mathbf{T}^{H} \mathbf{H}_{\mathrm{Tx}}^{H} \mathbf{H}_{\mathrm{Tx}} \mathbf{T}\right\} \leq P_{o}
$$

can be reformulated using the $\operatorname{vec}(\cdot)$ operator as $\left\|\operatorname{vec}\left(\mathbf{H}_{\mathrm{Tx}} \mathbf{T}\right)\right\| \leq \sqrt{P_{o}}$, which is equivalent to the SOC

$$
\left[\begin{array}{c}
\sqrt{P_{o}} \\
\operatorname{vec}\left(\mathbf{H}_{\mathrm{Tx}} \mathbf{T}\right)
\end{array}\right] \succeq_{K} 0
$$


Using (33) and (35), and denoting $p=\sqrt{P_{o}}$, the program (9) can be cast in the standard SOCP form [52]:

$$
\mathcal{P}\left(\gamma_{o}\right): \begin{cases}\min _{\mathbf{T}, p} \quad p \\
\text { s.t. } & {\left[\begin{array}{c}
\sqrt{1+\frac{1}{\gamma_{o}}}[\mathbf{H T}]_{i, i} \\
\mathbf{T}^{H} \mathbf{H}^{H} \mathbf{e}_{i} \\
\sigma_{i}
\end{array}\right] \succeq_{K} 0, \quad i=1, \cdots, K ;} \\
& {\left[\begin{array}{c}
p \\
\operatorname{vec}\left(\mathbf{H}_{\mathrm{Tx}} \mathbf{T}\right)
\end{array}\right] \succeq_{K} 0 .}\end{cases}
$$

Thus it can be efficiently solved using any standard SOCP package [51]. Such a solver can also numerically determine the feasibility of the optimization problem. A similar approach was taken in [42] in the context of transmit beamforming.

As explained in Section 3, each SOC constraint can be replaced with an SDP constraint using (14). Thus the problem can also be expressed as a standard SDP:

$$
\mathcal{P}\left(\gamma_{o}\right): \begin{cases}\min _{\mathbf{T}, p} & p \\ \text { s.t. } & \mathbf{A}_{i}(\mathbf{T}) \succeq 0, \quad i=1, \cdots, K ; \\ & \mathbf{C}(\mathbf{T}) \succeq 0,\end{cases}
$$

where

$$
\mathbf{A}_{i}(\mathbf{T})=\left[\begin{array}{cc}
\sqrt{1+\frac{1}{\gamma_{o}}}[\mathbf{H T}]_{i, i} & {\left[\begin{array}{cc}
\mathbf{e}_{i}^{H} \mathbf{H} \mathbf{T} & \sigma_{i}
\end{array}\right]} \\
{\left[\begin{array}{c}
\mathbf{T}^{H} \mathbf{H}^{H} \mathbf{e}_{i} \\
\sigma_{i}
\end{array}\right]} & \sqrt{1+\frac{1}{\gamma_{o}}}[\mathbf{H T}]_{i, i} \mathbf{I}
\end{array}\right], \quad i=1, \cdots, K
$$

and

$$
\mathbf{C}(\mathbf{T})=\left[\begin{array}{cc}
p & \operatorname{vec}^{H}\left(\mathbf{H}_{\mathrm{Tx}} \mathbf{T}\right) \\
\operatorname{vec}\left(\mathbf{H}_{\mathrm{Tx}} \mathbf{T}\right) & p \mathbf{I}
\end{array}\right]
$$

However, solving SOCPs via SDP is not very efficient. Interior point methods that solve SOCP directly have a much better worst case complexity than their SDP counterparts [52].

It is important to note that the above formulations are general and do not depend on the rank of the channel. Thus, these solutions are also appropriate for rank deficient channels. 


\subsection{OPTIMALITY CONDITIONS}

In this section, we will derive the KKT optimality conditions for the power optimization. As explained in the previous section, the problem can be numerically solved using standard optimization packages. Nonetheless, the conditions provide more insight into the solution. A simple structure based on the Lagrange dual variables is derived for the optimal solution. Given this structure, we propose two alternative methods for finding these variables. In Section 4.3.1, we derive a simple fixed point iteration which converges to these variables. The computational complexity of this approach is lower than that of the conic solution. Moreover, this solution does not require any external conic package which is not always available. Alternatively, in Section 4.3.2, we propose a dual SDP program, whose optimal arguments are the necessary variables. The main results are summarized in the following theorem:

Theorem 1. Consider the power optimization program $\mathcal{P}\left(\gamma_{o}\right)$ of (9). Define the dual variables $\lambda_{i}>0$ for $i=1 \cdots K$, and denote $\boldsymbol{\Lambda}=\operatorname{diag}\left\{\lambda_{i}\right\}$. If there exist $\lambda_{i}>0$ such that

$$
\gamma_{o}=\frac{1}{\frac{1}{\left[\boldsymbol{\Lambda}^{\frac{1}{2}} \mathbf{H}\left(\mathbf{H}^{H} \boldsymbol{\Lambda} \mathbf{H}+\mathbf{H}_{\mathrm{T} \mathbf{X}}^{H} \mathbf{H}_{\mathrm{TX}}\right)^{\dagger} \mathbf{H}^{H} \boldsymbol{\Lambda}^{\frac{1}{2}}\right]_{i, i}}-1}, \quad i=1, \cdots, K,
$$

holds, then the program is strictly feasible. Moreover, if (40) hold, then the optimal $\mathbf{T}$ is of the form

$$
\mathbf{T}=\left(\mathbf{H}^{H} \boldsymbol{\Lambda} \mathbf{H}+\mathbf{H}_{\mathrm{Tx}}^{H} \mathbf{H}_{\mathrm{Tx}}\right)^{\dagger} \mathbf{H}^{H} \boldsymbol{\Lambda}^{\frac{1}{2}} \operatorname{diag}\left\{\delta_{i}\right\}
$$

where $\delta_{i}$ are the positive weights that allocate the power between the users:

$$
\begin{aligned}
\delta_{i} & =\sqrt{\sum_{j}\left[\frac{\gamma_{o}}{1+\gamma_{o}} \mathbf{I}-\mathbf{F}\right]_{i, j}^{-1} \lambda_{j} \sigma_{j}^{2}}, \quad i=1, \cdots, K ; \\
{[\mathbf{F}]_{i, j} } & =\left[\boldsymbol{\Lambda}^{\frac{1}{2}} \mathbf{H}\left(\mathbf{H}^{H} \boldsymbol{\Lambda} \mathbf{H}+\mathbf{H}_{\mathrm{Tx}}^{H} \mathbf{H}_{\mathrm{Tx}}\right)^{\dagger} \mathbf{H}^{H} \boldsymbol{\Lambda}^{\frac{1}{2}}\right]_{i, j}^{2}, \quad i, j=1, \cdots, K .
\end{aligned}
$$

This structure of $\mathbf{T}$ is unique within the range of $\mathbf{H}_{\mathrm{Tx}}^{H}$. At this optimal solution, all the constraints are active, i.e., there are equal SINRs for all the subchannels. The optimal objective value is

$$
P_{o}=\sum_{i} \lambda_{i} \sigma_{i}^{2}
$$

Proof. The proof consists of two parts. First, we show that if (40) holds then the problem is strictly feasible. Next, assuming it is strictly feasible, we will use the KKT optimality conditions to show that the proposed 
solution is necessary and sufficient.

We begin by proving that if (40) holds, then the proposed solution in (41)-(43) is feasible. First, let us prove that this solution exists, i.e., that the matrix $\left[\frac{\gamma_{o}}{1+\gamma_{o}} \mathbf{I}-\mathbf{F}\right]$ in (42) is invertible and that the argument of the squared root is non negative. The matrix is invertible because that the maximal eigenvalue of $\mathbf{F}$ is less than $\frac{\gamma_{o}}{1+\gamma_{o}}$ :

$$
\begin{aligned}
\operatorname{eig}_{\max }(\mathbf{F}) \leq & \|\mathbf{F}\| \mid \\
= & \max _{i} \sum_{j}\left|\left[\boldsymbol{\Lambda}^{\frac{1}{2}} \mathbf{H}\left(\mathbf{H}^{H} \boldsymbol{\Lambda} \mathbf{H}+\mathbf{H}_{\mathrm{Tx}}^{H} \mathbf{H}_{\mathrm{Tx}}\right)^{\dagger} \mathbf{H}^{H} \boldsymbol{\Lambda}^{\frac{1}{2}}\right]_{i, j}^{2}\right| \\
= & \max _{i}\left[\boldsymbol{\Lambda}^{\frac{1}{2}} \mathbf{H}\left(\mathbf{H}^{H} \boldsymbol{\Lambda} \mathbf{H}+\mathbf{H}_{\mathrm{Tx}}^{H} \mathbf{H}_{\mathrm{Tx}}\right)^{\dagger} \mathbf{H}^{H} \boldsymbol{\Lambda} \mathbf{H}\left(\mathbf{H}^{H} \boldsymbol{\Lambda} \mathbf{H}+\mathbf{H}_{\mathrm{Tx}}^{H} \mathbf{H}_{\mathrm{Tx}}\right)^{\dagger} \mathbf{H}^{H} \boldsymbol{\Lambda}^{\frac{1}{2}}\right]_{i, i} \\
= & \max _{i}\left\{\left[\boldsymbol{\Lambda}^{\frac{1}{2}} \mathbf{H}\left(\mathbf{H}^{H} \boldsymbol{\Lambda} \mathbf{H}+\mathbf{H}_{\mathrm{Tx}}^{H} \mathbf{H}_{\mathrm{Tx}}\right)^{\dagger} \mathbf{H}^{H} \boldsymbol{\Lambda}^{\frac{1}{2}}\right]_{i, i}\right. \\
& \left.\quad-\left[\boldsymbol{\Lambda}^{\frac{1}{2}} \mathbf{H}\left(\mathbf{H}^{H} \boldsymbol{\Lambda} \mathbf{H}+\mathbf{H}_{\mathrm{Tx}}^{H} \mathbf{H}_{\mathrm{Tx}}\right)^{\dagger} \mathbf{H}_{\mathrm{Tx}}^{H} \mathbf{H}_{\mathrm{Tx}}\left(\mathbf{H}^{H} \boldsymbol{\Lambda} \mathbf{H}+\mathbf{H}_{\mathrm{Tx}}^{H} \mathbf{H}_{\mathrm{Tx}}\right)^{\dagger} \mathbf{H}^{H} \boldsymbol{\Lambda}^{\frac{1}{2}}\right]_{i, i}\right\} \\
\leq & \max _{i}\left\{\left[\boldsymbol{\Lambda}^{\frac{1}{2}} \mathbf{H}\left(\mathbf{H}^{H} \boldsymbol{\Lambda} \mathbf{H}+\mathbf{H}_{\mathrm{Tx}}^{H} \mathbf{H}_{\mathrm{Tx}}\right)^{\dagger} \mathbf{H}^{H} \boldsymbol{\Lambda}^{\frac{1}{2}}\right]_{i, i}\right\} \\
= & {\left[\mathbf{H}\left(\mathbf{H}^{H} \boldsymbol{\Lambda} \mathbf{H}+\mathbf{H}_{\mathrm{Tx}}^{H} \mathbf{H}_{\mathrm{Tx}}\right)^{\dagger} \mathbf{H}^{H} \boldsymbol{\Lambda}\right]_{i, i} } \\
= & \frac{\gamma_{o}}{1+\gamma_{o}},
\end{aligned}
$$

where the inequality in (45) stems from the fact that any induced matrix norm upper bounds the maximal eigenvalue of the matrix. The equality in (46) is the definition of the row sum induced matrix norm. The inequality in (49) stems from neglecting the non negative terms in (48), and the equality in (50) is due to (40). We still need to prove that the inequality is strict, but this can be proven as follows. Assume that the inequality is not strict, i.e., there exists an $i$ such that the second element in (48) is zero, i.e., $\mathbf{H}_{\mathrm{Tx}}\left(\mathbf{H}^{H} \boldsymbol{\Lambda} \mathbf{H}+\mathbf{H}_{\mathrm{Tx}}^{H} \mathbf{H}_{\mathrm{Tx}}\right)^{\dagger} \mathbf{H}^{H} \boldsymbol{\Lambda}^{\frac{1}{2}} \mathbf{e}_{i}=\mathbf{0}$, and therefore $\left[\boldsymbol{\Lambda}^{\frac{1}{2}} \mathbf{H}_{\mathrm{RxCh}} \mathbf{H}_{\mathrm{Tx}}\left(\mathbf{H}^{H} \boldsymbol{\Lambda} \mathbf{H}+\mathbf{H}_{\mathrm{Tx}}^{H} \mathbf{H}_{\mathrm{Tx}}\right)^{\dagger} \mathbf{H}^{H} \boldsymbol{\Lambda}^{\frac{1}{2}}\right]_{i, i}=$ 0. But, since $\gamma_{o}>0$, this a contradiction to (40), and therefore the inequality in (49) must be strict.

We now show that the arguments of the squared roots in (42) are non negative. Using a series expansion for the matrix inversion yields [55]

$$
\left[\begin{array}{c}
\delta_{1}^{2} \\
\vdots \\
\delta_{K}^{2}
\end{array}\right]=\left[\frac{\gamma_{o}}{1+\gamma_{o}} \mathbf{I}-\mathbf{F}\right]^{-1}\left[\begin{array}{c}
\lambda_{1} \sigma_{1}^{2} \\
\vdots \\
\lambda_{K} \sigma_{K}^{2}
\end{array}\right]=\frac{1+\gamma_{o}}{\gamma_{o}} \sum_{j=1}^{\infty}\left[\frac{\gamma_{o}}{1+\gamma_{o}} \mathbf{F}\right]^{j}\left[\begin{array}{c}
\lambda_{1} \sigma_{1}^{2} \\
\vdots \\
\lambda_{K} \sigma_{K}^{2}
\end{array}\right]
$$

The elements of $\frac{\gamma_{o}}{1+\gamma_{o}} \mathbf{F}$ are nonnegative. Therefore, the elements of the sum will also be non negative, and we can take the element wise squared roots and solve for $\delta_{i}$ for $i=1, \cdots, K$. 
Thus, we have shown that the solution in (41)-(43) exists. Plugging this solution into the SINR constraints satisfies all the constraints with equality. Therefore, the problem is feasible. Moreover, since $\sigma_{i}^{2}>0$ for $i=1, \cdots, K$, we can always scale the solution $\mathbf{T}$ by $c>1$, and satisfy the constraints with inequalities, i.e., the problem is strictly feasible.

In the next part of the proof, we will show that if (40) holds, then the solution in (41)-(43) is necessary and sufficient for optimality. The power optimization problem can be written as follows:

$$
\mathcal{P}\left(\gamma_{o}\right): \begin{cases}\min _{\mathbf{T}} & \operatorname{Tr}\left\{\mathbf{T}^{H} \mathbf{H}_{\mathrm{Tx}}^{H} \mathbf{H}_{\mathrm{Tx}} \mathbf{T}\right\} \\
\text { s.t. } & \left\|\left[\begin{array}{c}
\mathbf{T}^{H} \mathbf{H}^{H} \mathbf{e}_{i} \\
\sigma_{i}
\end{array}\right]\right\|^{2}-\left(1+\frac{1}{\gamma_{o}}\right)\left|[\mathbf{H T}]_{i, i}\right|^{2} \leq 0, \quad i=1, \cdots, K .\end{cases}
$$

The above program is not written in convex form (in order to write it in convex form, conic inequalities must be used). In general, the KKT conditions are not sufficient for optimality in non convex programs. However, in Appendix A, we show that in this special case, if the program is strictly feasible, then its KKT conditions are necessary and sufficient for optimality. The Lagrangian associated with program (53) is

$$
\begin{aligned}
\mathcal{L} & =\operatorname{Tr}\left\{\mathbf{T}^{H} \mathbf{H}_{\mathrm{Tx}}^{H} \mathbf{H}_{\mathrm{Tx}} \mathbf{T}\right\}+\sum_{i} \lambda_{i}\left[\left\|\left[\begin{array}{c}
\mathbf{T}^{H} \mathbf{H}^{H} \mathbf{e}_{i} \\
\sigma_{i}
\end{array}\right]\right\| \|^{2}-\left(1+\frac{1}{\gamma_{o}}\right)\left|[\mathbf{H} \mathbf{T}]_{i, i}\right|^{2}\right] \\
& =\operatorname{Tr}\left\{\mathbf{T}^{H}\left[\mathbf{H}^{H} \boldsymbol{\Lambda} \mathbf{H}+\mathbf{H}_{\mathrm{Tx}}^{H} \mathbf{H}_{\mathrm{Tx}}\right] \mathbf{T}\right\}+\sum_{i} \lambda_{i} \sigma_{i}^{2}-\sum_{i} \lambda_{i}\left(1+\frac{1}{\gamma_{o}}\right)\left|[\mathbf{H T}]_{i, i}\right|^{2},
\end{aligned}
$$

where $\lambda_{i} \geq 0$ are the Lagrange dual variables. As we have shown in the first part of the proof, if (40) holds, the problem is strictly feasible. Therefore, its primal and dual variables are optimal if and only if the following conditions are satisfied:

1. Feasibility: The variable $\mathbf{T}$ is feasible

$$
\left(1+\frac{1}{\gamma_{o}}\right)\left|[\mathbf{H T}]_{i, i}\right|^{2} \geq\left\|\left[\begin{array}{c}
\mathbf{T}^{H} \mathbf{H}^{H} \mathbf{e}_{i} \\
\sigma_{i}
\end{array}\right]\right\|^{2}, \quad i=1, \cdots, K,
$$

and the dual variables are dual feasible, i.e., $\lambda_{i} \geq 0$ for $i=1, \cdots, K$.

2. Complementary Slackness: For each $i=1, \cdots, K$, either $\lambda_{i}=0$ or

$$
\left(1+\frac{1}{\gamma_{o}}\right)\left|[\mathbf{H T}]_{i, i}\right|^{2}=\left\|\left[\begin{array}{c}
\mathbf{T}^{H} \mathbf{H}^{H} \mathbf{e}_{i} \\
\sigma_{i}
\end{array}\right]\right\|^{2}
$$


3. Zero derivative: The derivative of $\mathcal{L}$ with respect to $\mathbf{T}$ is zero, resulting in

$$
\left[\mathbf{H}^{H} \boldsymbol{\Lambda} \mathbf{H}+\mathbf{H}_{\mathrm{Tx}}^{H} \mathbf{H}_{\mathrm{Tx}}\right] \mathbf{T}=\mathbf{H}^{H} \boldsymbol{\Lambda}^{\frac{1}{2}} \operatorname{diag}\left\{\left(1+\frac{1}{\gamma_{o}}\right)\left[\boldsymbol{\Lambda}^{\frac{1}{2}} \mathbf{H} \mathbf{T}\right]_{i, i}\right\}
$$

At the optimal solution all the constraints are active, i.e., (56) holds with equality for $i=1, \cdots, K$. As proof, note that if one constraint does not hold with an equality, then we can always scale the row in $\mathbf{T}$ associated with it, and arrive with a feasible solution that results in a lower objective value, which is a contradiction.

Another important property of the optimal solution, is that all the dual variables are strictly positive. As proof, assume the contrary, i.e., there exists an $i$ such that $\lambda_{i}=0$. Then, multiplying (57) by $\mathbf{T}^{H}$ on the left and examining the $i$ 'th diagonal element, we have $\mathbf{e}_{i}^{H} \mathbf{T}^{H}\left[\mathbf{H}^{H} \mathbf{\Lambda} \mathbf{H}+\mathbf{H}_{\mathrm{Tx}}^{H} \mathbf{H}_{\mathrm{Tx}}\right] \mathbf{T e}_{i}=0$, which holds if and only if $\boldsymbol{\Lambda}^{\frac{1}{2}} \mathbf{H T} \mathbf{e}_{i}=\mathbf{0}$ and $\mathbf{H}_{\mathrm{Tx}} \mathbf{T} \mathbf{e}_{i}=\mathbf{0}$, in which case the $i$ 'th SINR is clearly zero. But, since $\gamma_{o}>0$, this contradicts the SINR constraints.

In general, the $\mathbf{T}$ that satisfies (57) is not unique. Nonetheless, expressing $\mathbf{T}$ as $\mathbf{T}=\mathbf{T}_{\|}+\mathbf{T}_{\perp}$ where $\mathbf{T}_{\|}=\mathbf{P T}, \mathbf{T}_{\perp}=(\mathbf{I}-\mathbf{P}) \mathbf{T}$, and $\mathbf{P}=\mathbf{H}_{\mathrm{Tx}}^{\dagger} \mathbf{H}_{\mathrm{Tx}}$, we can find $\mathbf{T}_{\|}$which is unique within the range of $\mathbf{H}_{\mathrm{Tx}}^{H}$. Using $\mathbf{H T}=\mathbf{H T}_{\|}$, we arrive with the following necessary and sufficient conditions:

$$
\begin{gathered}
\mathbf{T}_{\|}=\left[\mathbf{H}^{H} \boldsymbol{\Lambda} \mathbf{H}+\mathbf{H}_{\mathrm{Tx}}^{H} \mathbf{H}_{\mathrm{Tx}}\right]^{\dagger} \mathbf{H}^{H} \boldsymbol{\Lambda}^{\frac{1}{2}} \operatorname{diag}\left\{\delta_{i}\right\} ; \\
\left(1+\frac{1}{\gamma_{o}}\right)\left|\left[\mathbf{H} \mathbf{T}_{\|}\right]_{i, i}\right|^{2}=\left\|\left[\begin{array}{c}
\mathbf{T}_{\|}^{H} \mathbf{H}^{H} \mathbf{e}_{i} \\
\sigma_{i}
\end{array}\right]\right\|^{2}, \quad i=1, \cdots, K,
\end{gathered}
$$

where $\delta_{i}=\left(1+\frac{1}{\gamma_{o}}\right)\left[\boldsymbol{\Lambda}^{\frac{1}{2}} \mathbf{H} \mathbf{T}_{\|}\right]_{i, i}$ for $i=1, \cdots, K$. As already explained, if (40) holds then the solution in (41)-(43) satisfies (59). In addition, it has the structure of (58) and is therefore sufficient. Moreover, it is easy to show that this structure is also necessary (within the range of $\mathbf{H}_{\mathrm{Tx}}^{H}$ ). Plugging $\mathbf{T}_{\|}$from (58) into (59) yields

$$
\left(1+\frac{1}{\gamma_{o}}\right) \frac{1}{\lambda_{i}}[\mathbf{F}]_{i, i} \delta_{i}^{2}=\sum_{j} \frac{1}{\lambda_{i}}[\mathbf{F}]_{i, j} \delta_{j}^{2}+\sigma_{i}^{2}, \quad i=1, \cdots K
$$


where $\mathbf{F}$ is the matrix defined by (43). Rewriting in matrix form, we have

$$
\left[\frac{\gamma_{o}}{1+\gamma_{o}} \mathbf{I}-\mathbf{F}\right]\left[\begin{array}{c}
\delta_{1}^{2} \\
\vdots \\
\delta_{K}^{2}
\end{array}\right]=\left[\begin{array}{c}
\lambda_{1} \sigma_{1}^{2} \\
\vdots \\
\lambda_{K} \sigma_{K}^{2}
\end{array}\right]
$$

Since $[\mathbf{H T}]_{i, i} \geq 0$ for $i=1, \cdots, K$, the unique solution to this set of equations in given by (42)-(43). Finally, the optimal objective value in (44) can be easily found using (8) and (57).

Theorem 1 provides a simple strategy for designing the precoder. Given a feasible $\gamma_{o}$, all one has to do is find $\lambda_{i}>0$ which satisfy (40). Once these are found, $\mathbf{T}$ can be derived through (41)-(43). As we will show in Section 6, in some special cases, these variables can be derived in closed form. Otherwise, we now propose two alternative methods for finding these variables. In Section 4.3.1, we present a simple fixed point iteration, and in Section 4.3.2 we propose the use a SDP dual program.

\subsubsection{FIXED POINT ITERATION FOR FINDING $\lambda_{i}$}

The structure of (40) motivates a fixed point iteration for finding $\lambda_{i}$. By rearranging (40), we arrive with the following simple iteration:

$$
\lambda_{i}^{(n+1)}=\frac{\gamma_{o}}{1+\gamma_{o}} \frac{1}{\left[\mathbf{H}\left(\mathbf{H}^{H} \operatorname{diag}\left\{\lambda_{i}^{(n)}\right\} \mathbf{H}+\mathbf{H}_{\mathrm{Tx}}^{H} \mathbf{H}_{\mathrm{Tx}}\right)^{\dagger} \mathbf{H}^{H}\right]_{i, i}}, \quad i=1, \cdots K .
$$

Clearly, the optimal $\lambda_{i}$ satisfy this fixed point. As we now show, if $\mathcal{P}\left(\gamma_{o}\right)$ is feasible, then the above iteration will converge from any $\lambda_{i}^{(0)}$ to a set $\lambda_{i}^{(n)}>0$ that satisfies (40). The convergence proof is based on the standard function approach introduced in [56], which can be summarized as follows. Consider the fixed point iteration

$$
\lambda_{i}^{(n+1)}=f_{i}\left(\boldsymbol{\Lambda}^{(n)}\right), \quad i=1, \cdots K
$$

where $\boldsymbol{\Lambda}^{(n)}=\operatorname{diag}\left\{\lambda^{(n)}\right\}$. If the functions $f_{i}(\boldsymbol{\Lambda})$ obey the following properties:

- Positivity $f_{i}(\boldsymbol{\Lambda})>0$ for all $i$;

- Monotonicity If $\lambda_{i} \geq \lambda_{i}^{\prime}$ for all $i$, then $f_{i}(\boldsymbol{\Lambda}) \geq f_{i}\left(\boldsymbol{\Lambda}^{\prime}\right)$ for all $i$;

- Scalability If $\alpha>1$, then $\alpha f_{i}(\boldsymbol{\Lambda})>f_{i}(\alpha \boldsymbol{\Lambda})$ for all $i$, 


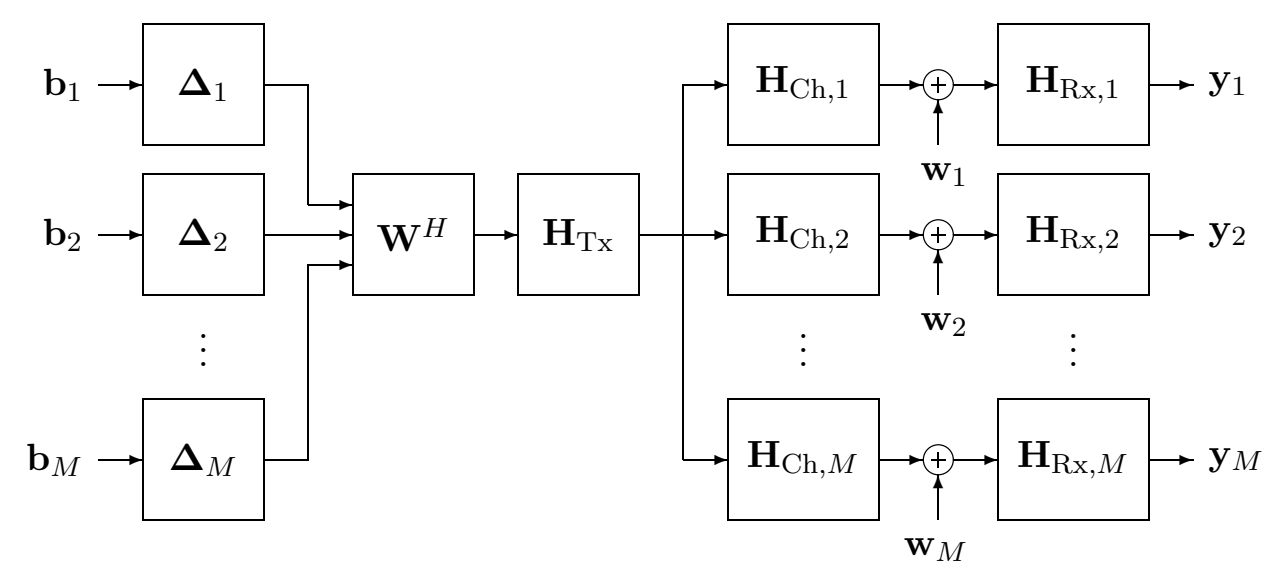

Figure 2: Block diagram of a downlink (broadcast) system. The matrices $\boldsymbol{\Delta}_{m}$ for $m=1, \cdots, M$, are diagonal matrices with the $\delta_{i}$ s associated with $\mathbf{b}_{m}$.

then the iteration has a fixed point, it is unique, and for any initial $\boldsymbol{\Lambda}^{(0)}$, the iteration will converge to it. In Appendix B, we show that if the problem is feasible, and $\left[\mathbf{H}\left(\mathbf{H}_{\mathrm{Tx}}^{H} \mathbf{H}_{\mathrm{Tx}}\right)^{\dagger} \mathbf{H}^{H}\right]_{i, i}<\infty$ for $i=1, \cdots, K$, then the functions in (62) satisfy these exact properties. Thus, the iteration will converge.

\subsubsection{DUAL PROGRAM FOR FINDING $\lambda_{i}$}

Alternatively, the dual variables can be found through a dual program. The dual program is a concave program that optimizes the dual variables. The details of its derivation are provided in Appendix C. The resulting program is:

$$
\mathcal{P}_{\mathcal{D}}\left(\gamma_{o}\right): \begin{cases}\max _{\lambda_{i} \geq 0} & \sum_{i} \lambda_{i} \sigma_{i}^{2} \\ \text { s.t. } & \frac{\gamma_{o}}{1+\gamma_{o}}\left[\mathbf{H}^{H} \mathbf{\Lambda} \mathbf{H}+\mathbf{H}_{\mathrm{Tx}}^{H} \mathbf{H}_{\mathrm{Tx}}\right]-\lambda_{i} \mathbf{H}^{H} \mathbf{e}_{i} \mathbf{e}_{i}^{H} \mathbf{H} \succeq 0, \quad i=1, \cdots, K .\end{cases}
$$

This is a simple SDP/LMI program, which can be efficiently solved by any standard SDP/LMI optimization package. Moreover, it has only $K$ optimization variables, in comparison to $K^{2}$ optimization variables in the original program, and therefore has a lower computational complexity. A similar result was obtained in [42] in the context of beamforming. 


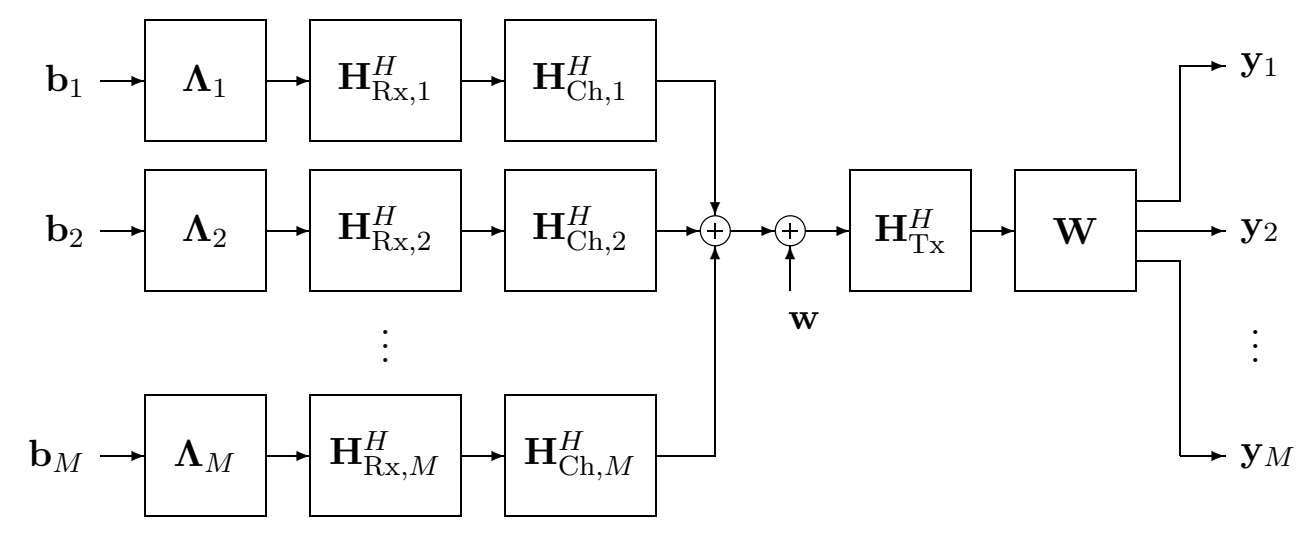

Figure 3: Block diagram of a uplink (multiple access) system. The matrices $\boldsymbol{\Lambda}_{m}$ for $m=1, \cdots, M$, are diagonal matrices with the $\lambda_{i}$ s associated with $\mathbf{b}_{m}$. The vector $\mathbf{w}$ is the virtual uplink noise vector.

\subsection{INTERPRETATION USING DOWNLINK UPLINK DUALITY}

In this section, we provide an alternative solution for the power optimization problem based on the well known downlink uplink duality [43,44]. As explained in the previous sections, the power optimization can be solved efficiently without the use of duality. However, previous attempts for solving the downlink beamforming problem, which is a special case of precoding (where $\mathbf{H}_{\mathrm{Tx}}=\mathbf{I}$ ), are based on this approach. Therefore, for completeness, we now review this method and generalize it to the case of precoding, i.e., arbitrary $\mathbf{H}_{\mathrm{Tx}}$. Moreover, the duality is interesting from an engineering point on view, as it provides an interesting physical interpretation for the solution.

Recently, an interesting duality was found between downlink beamforming and another problem called uplink beamforming. It is usually referred to as downlink (broadcast) - uplink (multiple access) duality, since one problem is usually found in the broadcast channel of a downlink system, and the other is found in the multiple access channel of an uplink system. Fortunately, the uplink beamforming problem is easier to solve. Using the duality, the downlink solution can be derived through the uplink solution. For simplicity, in the sequel, we restrict ourselves to full rank channels (as did all the works in this context). Mathematically, the duality can be stated as follows: 
Theorem 2. Consider the downlink program $\mathcal{P}$ :

$$
\mathcal{P}\left(\gamma_{o}\right)= \begin{cases}\min _{\mathbf{T}} & \operatorname{Tr}\left\{\mathbf{T}^{H} \mathbf{H}_{\mathrm{Tx}}^{H} \mathbf{H}_{\mathrm{Tx}} \mathbf{T}\right\} \\ \text { s.t. } & \frac{\left|[\mathbf{H T}]_{i, i}\right|^{2}}{\sum_{j \neq i}\left|[\mathbf{H T}]_{i, j}\right|^{2}+\sigma_{i}^{2}} \geq \gamma_{o}, \quad i=1, \cdots, K,\end{cases}
$$

and the uplink program:

$$
\overline{\mathcal{P}}\left(\gamma_{o}\right)= \begin{cases}\min _{\lambda_{i}>0} & \sum_{i} \sigma_{i}^{2} \lambda_{i} \\ \text { s.t. } & \frac{\lambda_{i}\left|\left[\mathbf{W} \mathbf{H}^{H}\right]_{i, i}\right|^{2}}{\sum_{j \neq i} \lambda_{j}\left|\left[\mathbf{W} \mathbf{H}^{H}\right]_{i, j}\right|^{2}+\left[\mathbf{W} \mathbf{H}_{\mathrm{Tx}}^{H} \mathbf{H}_{\mathrm{Tx}} \mathbf{W}^{H}\right]_{i, i}} \geq \gamma_{o}, \quad i=1, \cdots, K .\end{cases}
$$

If the optimal arguments and objective value of $\overline{\mathcal{P}}$ are $\mathbf{W}, \lambda_{i}$, and $P_{o}$, then the optimal objective value of $\mathcal{P}$ is also $P_{o}$, and its optimal argument is $\mathbf{T}=\mathbf{W}^{H} \boldsymbol{\Lambda}^{\frac{1}{2}} \operatorname{diag}\left\{\delta_{i}\right\}$, where $\delta_{i}$ are defined in Theorem 1.

Proof. It is easy to see that each constraint in $\overline{\mathcal{P}}$ deals with one row of $\mathbf{W}$ and that the objective is not a function of $\mathbf{W}$ at all. Therefore, it is clear that each row of $\mathbf{W}$ will be chosen to maximize the SINR associated with it. Thus, for fixed $\lambda_{i}$, the optimal receiver $\mathbf{W}$ is the well known MMSE matrix [3]:

$$
\mathbf{W}=\mathbf{H}\left(\mathbf{H}^{H} \operatorname{diag}\left\{\lambda_{i}\right\} \mathbf{H}+\mathbf{H}_{\mathrm{Tx}}^{H} \mathbf{H}_{\mathrm{Tx}}\right)^{-1}
$$

which is unique up to a diagonal matrix multiplication on the left. In addition, similarly to the downlink problem, all the constraints of the uplink problem are active (otherwise, one can always decrease the $\lambda_{i}$ associated with the passive constraint and decrease the objective). Thus, at the optimum:

$$
\frac{\lambda_{i}\left|\left[\mathbf{W H}^{H}\right]_{i, i}\right|^{2}}{\sum_{j \neq i} \lambda_{j}\left|\left[\mathbf{W} \mathbf{H}^{H}\right]_{i, j}\right|^{2}+\left[\mathbf{W H}_{\mathrm{Tx}}^{H} \mathbf{H}_{\mathrm{Tx}} \mathbf{W}^{H}\right]_{i, i}}=\gamma_{o}, \quad i=1, \cdots, K .
$$

Plugging in the optimal $\mathbf{W}$ and simplifying the terms results in

$$
\lambda_{i}\left[\mathbf{H}\left(\mathbf{H}^{H} \operatorname{diag}\left\{\lambda_{i}\right\} \mathbf{H}+\mathbf{H}_{\mathrm{Tx}}^{H} \mathbf{H}_{\mathrm{Tx}}\right)^{-1} \mathbf{H}^{H}\right]_{i, i}=\frac{\gamma_{o}}{1+\gamma_{o}}, \quad i=1, \cdots, K
$$

Thus, the optimal $\lambda_{i}$ 's of $\overline{\mathcal{P}}$ satisfy (40). Due to (67), the precoder $\mathbf{T}=\mathbf{W}^{H} \boldsymbol{\Lambda}^{\frac{1}{2}} \operatorname{diag}\left\{\delta_{i}\right\}$ satisfies also (41). Therefore, according to Theorem 1 , this precoder is optimal for $\mathcal{P}$.

This uplink downlink duality was developed in [43] for the special case of $\mathbf{H}_{\mathrm{Tx}}=\mathbf{I}$. In Theorem 2 we generalize this result to arbitrary $\mathbf{H}_{\mathrm{Tx}}$. The importance of this theorem is in its interesting interpretation of the optimal solution and to the duality between the downlink and the uplink problem. For example, it 
provides a physical interpretation to the positive dual variables $\lambda_{i}>0$ as the virtual power allocation. In order to visualize this duality, we provide block diagrams of the two dual systems in Figs. 2 and 3.

Moreover, previous attempts for solving the $\mathcal{P}$ problem are based on this duality. As we have shown in the previous section, the problem can be solved without the duality using the optimality conditions. However, for completeness, we now present the duality based approach as well. This approach confronts the $\mathcal{P}$ problem by addressing the $\overline{\mathcal{P}}$ problem first and then adjusting the solution based on Theorem 2 . Fortunately, there is an intuitive iterative solution to program $\overline{\mathcal{P}}$. The problem can be solved by iteratively solving for each of the parameters, while keeping the others fixed $[41,46]$ :

$$
\begin{aligned}
& \overline{\mathcal{P}}\left(\gamma_{o}\right) \\
& 1 \text { repeat } \\
& 2 \\
& \text { for } i \leftarrow 1 \text { to } K \\
& 3 \\
& \mathbf{w}_{i} \leftarrow \arg \left\{\max _{\mathbf{w}_{i}} \frac{\lambda_{i}\left|\mathbf{w}_{i}^{H} \mathbf{H}^{H} \mathbf{e}_{i}\right|^{2}}{\sum_{j \neq i} \lambda_{j}\left|\mathbf{w}_{i} \mathbf{H}^{H} \mathbf{e}_{j}\right|^{2}+\left\|\mathbf{H}_{\mathrm{Tx}} \mathbf{w}_{i}\right\|^{2}}\right\} \\
& 4 \quad \boldsymbol{\Lambda} \leftarrow \arg \left\{\begin{array}{cl}
\min _{\lambda_{i}} & \sum_{i} \sigma_{i}^{2} \lambda_{i} \\
\text { s.t. } & \frac{\lambda_{i}\left|\mathbf{w}_{i}^{H} \mathbf{H}^{H} \mathbf{e}_{i}\right|^{2}}{\sum_{j \neq i} \lambda_{j}\left|\mathbf{w}_{i} \mathbf{H}^{H} \mathbf{e}_{j}\right|^{2}+\left\|\mathbf{H}_{\mathrm{Tx}} \mathbf{w}_{i}\right\|^{2}} \geq \gamma_{o}, \quad i=1, \cdots, K .
\end{array}\right\}
\end{aligned}
$$

where $\mathbf{w}_{i}^{H}$ is the $i$ th row of $\mathbf{W}$. Each optimization in line 3 tries to maximize an SINR using a receive weight vector. Their optimal solutions are given by the columns of (67). The optimization in line 4 can be carried out using the following fixed point iteration [56]:

$$
\lambda_{i} \leftarrow \gamma_{o} \frac{\sum_{j \neq i} \lambda_{j}\left|\mathbf{w}_{i} \mathbf{H}^{H} \mathbf{e}_{j}\right|^{2}+\left\|\mathbf{H}_{\mathrm{Tx}} \mathbf{w}_{i}\right\|^{2}}{\left|\mathbf{w}_{i}^{H} \mathbf{H}^{H} \mathbf{e}_{i}\right|^{2}}, \quad i=1, \cdots, K .
$$

In [46] it was shown that the above algorithm using (70) always converges to the optimal solution. However, the algorithm is quite complicated, and consists of an outer iteration with two inner iterations. Therefore, it is not as appealing as our simple fixed point iteration in (62).

\section{SINR OPTIMIZATION}

We now consider the problem of maximizing the worst SINR subject to a power constraint, i.e., the $\mathcal{S}$ program of (10). As before, we begin by examining its feasibility. Fortunately, it is easy to verify that the $\mathcal{S}$ program is always feasible, as we can always scale $\mathbf{T}$ so that it would satisfy the power constraint. In Section 5.1, we discuss the connection between the power optimization and the SIR optimization and explain how this connection can be used to solve the SIR optimization. Then, we follow the steps we took before in the 
context of the power optimization, and repeat them in the context of the SINR optimization. In Section 5.2, we formulate the SIR problem as a standard GEVP conic program, in Section 5.3 we provide a fixed point iteration, and in Section 5.4 we discuss its downlink uplink duality.

\subsection{CONNECTION WITH POWER OPTIMIZATION}

The most interesting property of the SINR optimization program is its relation to the power optimization program. In order to mathematically define this relation, we introduce the following theorem:

Theorem 3. The power optimization problem of (9) and the SINR optimization problem of (10) are inverse problems:

$$
\begin{aligned}
\gamma_{o} & =\mathcal{S}\left(\mathcal{P}\left(\gamma_{o}\right)\right) \\
P_{o} & =\mathcal{P}\left(\mathcal{S}\left(P_{o}\right)\right) .
\end{aligned}
$$

In addition, the optimal objective value of each program is continuous, and strictly monotonic increasing in its input argument:

$$
\begin{array}{lll}
\gamma_{o}>\widetilde{\gamma}_{o} & \Rightarrow & \mathcal{P}\left(\gamma_{o}\right)>\mathcal{P}\left(\widetilde{\gamma}_{o}\right) \\
P_{o}>\widetilde{P}_{o} & \Rightarrow & \mathcal{S}\left(P_{o}\right)>\mathcal{S}\left(\widetilde{P}_{o}\right) .
\end{array}
$$

Proof. We begin by proving (71) by contradiction. Assume the contrary, i.e. $P$ and $\mathbf{T}$ are the optimal value and argument of $\mathcal{P}(\gamma)$, and $\widetilde{\gamma} \neq \gamma$ and $\widetilde{\mathbf{T}}$ are the optimal value and argument of $\mathcal{S}(P)$. If $\widetilde{\gamma}<\gamma$, then this is a contradiction for the optimality of $\widetilde{\mathbf{T}}$ for $\mathcal{S}(P)$, since $\mathbf{T}$ is feasible for it, and provides a larger objective value $\gamma$. Otherwise, if $\widetilde{\gamma}>\gamma$, then this is a contradiction for the optimality of $\mathbf{T}$ for $\mathcal{P}(\gamma)$, since $\widetilde{\gamma}>\gamma$, and we can always find $c<1$ such that $c \widetilde{\mathbf{T}}$ will still be feasible, but will result in a smaller objective.

Next, we prove (73) by contradiction. Assume the contrary, i.e., $P$ and $\mathbf{T}$ are optimal for $\gamma$, and $\widetilde{P} \geq P$ and $\widetilde{\mathbf{T}}$ are optimal for $\widetilde{\gamma}<\gamma$. We can always multiply $\mathbf{T}$ by $c<1$ so that it will still achieve the SINRs constraints of $\widetilde{\gamma}$, with an effective power constraint $c^{2} P<P \leq \widetilde{P}$. This contradicts the assumption that $\widetilde{\mathbf{T}}$ was optimal for $\widetilde{\gamma}$. The continuity can be verified using similar arguments to those in Lemma 2 of [57]. The proofs of (72) and (74) are similar and are therefore omitted.

Using the properties in the Theorem 3, we can solve $\mathcal{S}\left(P_{o}\right)$ for a given $P_{o}$ by iteratively solving $\mathcal{P}\left(\gamma_{o}\right)$ for different $\gamma_{o}$ 's. Due to the inversion property, if $P_{o}=\mathcal{P}\left(\gamma_{o}\right)$, then its solution will be optimal also for 
$\mathcal{S}\left(P_{o}\right)$. The strict monotonicity and continuity guarantees that a simple one dimensional bisection search will efficiently find the required $\gamma_{o}$. This procedure is summarized in the following algorithm (see also [47]):

$$
\begin{array}{rcr}
\mathcal{S}\left(P_{o}\right) & \\
1 & \gamma_{\max } \leftarrow \operatorname{MaxSINR} \\
2 & \gamma_{\min } \leftarrow \text { MinSINR } \\
3 & \text { repeat } \\
4 & \gamma_{o} \leftarrow\left(\gamma_{\min }+\gamma_{\max }\right) / 2 \\
5 & \widehat{P_{o}} \leftarrow \mathcal{P}\left(\gamma_{o}\right) \\
6 & \text { if } \widehat{P_{o}} \leq P_{o} \\
7 & \text { then } \gamma_{\min } \leftarrow \gamma_{o} \\
8 & \text { else } \gamma_{\max } \leftarrow \gamma_{o} \\
9 & \text { until } \widehat{P_{o}}=P_{o} \\
10 & \text { return } \gamma_{o}
\end{array}
$$

where MinSINR and MaxSINR define a range of relevant SINRs for a specific application, and where we have used the convention that $\infty=\mathcal{P}\left(\gamma_{o}\right)$ if it is infeasible.

Theoretically, this means that the SINR optimization problem can be solved through the previous results concerning the power optimization. Nonetheless, due to its importance and in order to obtain more efficient numerical solution, we now provide direct solutions for the SINR optimization through conic optimization, via the optimality conditions, and through the downlink uplink duality.

\subsection{CONIC OPTIMIZATION SOLUTION}

The SINR optimization can be cast as a standard conic program. Using a slack variable, the problem can be rewritten as

$$
\mathcal{S}\left(P_{o}\right): \begin{cases}\max _{\mathbf{T}, \gamma_{o}} & \gamma_{o} \\ \text { s.t. } & \frac{\mid \mathbf{H} \mathbf{T}]\left._{i, i}\right|^{2}}{\sum_{i \neq j}\left|[\mathbf{H T}]_{i, j}\right|^{2}+\sigma_{i}^{2}} \geq \gamma_{o}, \quad i=1, \cdots, K ; \\ & \operatorname{Tr}\left\{\mathbf{T}^{H} \mathbf{H}_{\mathrm{Tx}}^{H} \mathbf{H}_{\mathrm{Tx}} \mathbf{T}\right\} \leq P_{o} .\end{cases}
$$

At first glance, (75) seems similar to (29). However, it turns out to be considerably more complicated. This is because the SINR matrix inequalities in (38) are linear in $\beta=\sqrt{1+\frac{1}{\gamma_{o}}}$ or in $\mathbf{T}$, but not in both simultaneously. Thus, when $\beta$ is an optimization variable and not a parameter, these constraints are no 
longer LMIs. In fact, the sets which they define are not convex ${ }^{2}$. Nonetheless, we can still express them using generalized matrix inequalities as in (37) and (38). If we rewrite the $\mathbf{A}_{i}(\mathbf{T})$ 's in (38) and separate out the terms which are linear, we have

$$
\mathbf{A}_{i}(\mathbf{T})=\beta \mathbf{A}_{i}^{1}(\mathbf{T})-\mathbf{A}_{i}^{2}(\mathbf{T})
$$

where $\mathbf{A}_{i}^{1}(\mathbf{T})$ and $\mathbf{A}_{i}^{2}(\mathbf{T})$ are matrices that depend affinely on $\mathbf{T}$ :

$$
\begin{aligned}
& \mathbf{A}_{i}^{1}(\mathbf{T})=\left[\begin{array}{cc}
{[\mathbf{H T}]_{i, i}} & \mathbf{0} \\
\mathbf{0} & {[\mathbf{H T}]_{i, i} \mathbf{I}}
\end{array}\right] \\
& \left.\mathbf{A}_{i}^{2}(\mathbf{T})=\left[-\left[\begin{array}{c}
0 \\
\mathbf{T}^{H} \mathbf{H}^{H} \mathbf{e}_{i} \\
\sigma_{i}
\end{array}\right] \begin{array}{cc}
-\left[\mathbf{e}_{i}^{H} \mathbf{H T}\right. & \sigma_{i}
\end{array}\right]\right] .
\end{aligned}
$$

Using (76) we can express $\mathcal{S}$ in the standard GEVP form:

$$
\mathcal{S}\left(P_{o}\right): \begin{cases}\min _{\mathbf{T}, \beta} & \beta \\ \text { s.t. } \quad & \beta \mathbf{A}_{i}^{1}(\mathbf{T}) \succeq \mathbf{A}_{i}^{2}(\mathbf{T}), \quad i=1, \cdots, K ; \\ & \mathbf{A}_{i}^{1}(\mathbf{T}) \succeq 0, \quad i=1, \cdots, K ; \\ & \mathbf{C}(\mathbf{T}) \succeq 0 .\end{cases}
$$

It can be solved using appropriate software, e.g., the GEVP command in the LMI toolbox [58].

As before, it is important to note that the GEVP formulation is general and does not depend on the rank of the channel. Thus, this solutions is also appropriate for rank deficient channels.

\subsection{A FIXED POINT ITERATION FOR FINDING $\lambda_{i}$}

The SINR optimization problem can also be solved using the conditions in Theorem 1. As explained in Theorem 3, $\mathcal{S}$ and $\mathcal{P}$ are inverse problems. Thus, the optimal solution of the SINR optimization is also optimal for an inverse power optimization problem, and therefore must satisfy its optimality conditions as well. All we need to do in order to solve the SINR optimization is find $\lambda_{i}>0$ that satisfy (40) and (44). Unfortunately, in this case, $\gamma_{o}$ is an optimization variable and not a parameter and has to be found as well.

\footnotetext{
${ }^{2}$ The exact definition of such sets is quasi convex [18].
} 
This can be overcome by adjusting the fixed point iteration in (62):

$$
\widetilde{\lambda}_{i}=\frac{1}{\left[\mathbf{H}\left(\mathbf{H}^{H} \operatorname{diag}\left\{\lambda_{i}^{(n)}\right\} \mathbf{H}+\mathbf{H}_{\mathrm{Tx}}^{H} \mathbf{H}_{\mathrm{Tx}}\right)^{\dagger} \mathbf{H}^{H}\right]_{i, i}}, \quad i=1, \cdots, K,
$$

and then normalizing the result so that it will satisfy (44):

$$
\lambda_{i}^{(n+1)}=\frac{P_{o} \widetilde{\lambda}_{i}}{\sum_{j} \sigma_{j}^{2} \widetilde{\lambda}_{j}}, \quad i=1, \cdots K
$$

If this iteration converges to a fixed point $\lambda_{i}^{(n)}>0$ then it will satisfy (40) and (44). We do not have a convergence proof for this iteration. However, numerous numerical simulations with arbitrary initial points and parameters show a rapid convergence rate.

\subsection{INTERPRETATION USING DOWNLINK UPLINK DUALITY}

Following the success of the uplink downlink duality in the power optimization, the duality was recently used to confront the SINR optimization $[48,49]$. The uplink downlink duality in the case of the SINR optimization can be stated as follows:

Theorem 4. Recall the downlink program $\mathcal{S}$ :

$$
\mathcal{S}\left(P_{o}\right)= \begin{cases}\max _{\mathbf{T}, \gamma_{o}} & \gamma_{o} \\ \text { s.t. } & \frac{\left|[\mathbf{H T}]_{i, i}\right|^{2}}{\sum_{j \neq i}\left|[\mathbf{H T}]_{i, j}\right|^{2}+\sigma_{i}^{2}} \geq \gamma_{o}, \quad i=1, \cdots, K \\ & \operatorname{Tr}\left\{\mathbf{T}^{H} \mathbf{H}_{\mathrm{Tx}}^{H} \mathbf{H}_{\mathrm{Tx}} \mathbf{T}\right\} \leq P_{o}\end{cases}
$$

and consider the following uplink program:

$$
\overline{\mathcal{S}}\left(P_{o}\right)= \begin{cases}\max _{\mathbf{W}, \lambda_{i} \geq 0, \gamma_{o}} & \gamma_{o} \\ \text { s.t. } & \frac{\lambda_{i}\left|\left[\mathbf{W} \mathbf{H}^{H}\right]_{i, i}\right|^{2}}{\sum_{j \neq i} \lambda_{j}\left|\left[\mathbf{W} \mathbf{H}^{H}\right]_{i, j}\right|^{2}+\left[\mathbf{W H}_{\mathrm{Tx}}^{H} \mathbf{H}_{\mathrm{Tx}} \mathbf{W}^{H}\right]_{i, i}} \geq \gamma_{o}, \quad i=1, \cdots, K ; \\ & \sum_{i} \sigma_{i}^{2} \lambda_{i} \leq P_{o} .\end{cases}
$$

If the optimal arguments and objective value of $\overline{\mathcal{S}}$ are $\mathbf{W}, \lambda_{i}$, and $\gamma_{o}$, then the optimal objective value of $\mathcal{S}$ is also $\gamma_{o}$, and its optimal argument is $\mathbf{T}=\mathbf{W}^{H} \boldsymbol{\Lambda}^{\frac{1}{2}} \operatorname{diag}\left\{\delta_{i}\right\}$, where $\delta_{i}$ are non negative weights.

Proof. The proof is similar to the other proofs in this paper, and therefore we only provide a sketch of it. As before, the problems $\overline{\mathcal{P}}$ and $\overline{\mathcal{S}}$ are inverse problems. But in Theorem 2 we have shown that $\mathcal{P}$ and $\overline{\mathcal{P}}$ 
are equivalent. Therefore, $\mathcal{S}$ and $\overline{\mathcal{S}}$ must be equivalent too. A more detailed proof on this duality in the case of $\mathbf{H}_{\mathrm{Tx}}=\mathbf{I}$ can be found in [49].

As explained, the downlink beamforming SINR optimization problem was solved using this duality $[48,49]$. As before, the algorithm iteratively optimizes each of the optimization variables while keeping the others fixed:

$$
\begin{aligned}
& \overline{\mathcal{S}}\left(P_{o}\right) \\
& 1 \text { repeat } \\
& 2 \quad \text { for } i \leftarrow 1 \text { to } K \\
& 3 \quad \mathbf{w}_{i} \leftarrow \arg \left\{\max _{\mathbf{w}_{i}} \frac{\lambda_{i}\left|\mathbf{w}_{i}^{H} \mathbf{H}^{H} \mathbf{e}_{i}\right|^{2}}{\sum_{j \neq i} \lambda_{j}\left|\mathbf{w}_{i} \mathbf{H}^{H} \mathbf{e}_{j}\right|^{2}+\left\|\mathbf{H}_{\mathrm{Tx}} \mathbf{w}_{i}\right\|^{2}}\right\} \\
& 4\left\{\gamma_{o}, \boldsymbol{\Lambda}\right\} \leftarrow \arg \left\{\begin{array}{ll}
\max _{\lambda_{i}, \gamma_{o}} & \gamma_{o} \\
\text { s.t. } & \frac{\lambda_{i}\left|\mathbf{w}_{i}^{H} \mathbf{H}^{H} \mathbf{e}_{i}\right|^{2}}{\sum_{j \neq i} \lambda_{j}\left|\mathbf{w}_{i} \mathbf{H}^{H} \mathbf{e}_{j}\right|^{2}+\left\|\mathbf{H}_{\mathrm{Tx}} \mathbf{w}_{i}\right\|^{2}} \geq \gamma_{o}, \quad i=1, \cdots, K ; \\
& \sum_{i} \sigma_{i}^{2} \lambda_{i} \leq P_{o} .
\end{array}\right\} \\
& 5 \text { until convergence }
\end{aligned}
$$

The solution for the optimization in line 3 remains as in (67). The optimization in line 4 is more complicated. It is based on the work of [13], which showed that this optimization can be carried out using eigenvalue optimization. As already discussed, at the optimal solution, all the constraints are active:

$$
\frac{\lambda_{i}\left|\mathbf{w}_{i}^{H} \mathbf{H}^{H} \mathbf{e}_{i}\right|^{2}}{\sum_{j \neq i} \lambda_{j}\left|\mathbf{w}_{i} \mathbf{H}^{H} \mathbf{e}_{j}\right|^{2}+\left\|\mathbf{H}_{\mathrm{Tx}} \mathbf{w}_{i}\right\|^{2}}=\gamma_{o}, \quad i=1, \cdots, K
$$

or

$$
\left(1+\frac{1}{\gamma_{o}}\right) \lambda_{i}=\sum_{j} \frac{\left|\mathbf{w}_{i} \mathbf{H}^{H} \mathbf{e}_{j}\right|^{2}}{\left|\mathbf{w}_{i}^{H} \mathbf{H}^{H} \mathbf{e}_{i}\right|^{2}} \lambda_{j}+\frac{\left\|\mathbf{H}_{\mathrm{Tx}} \mathbf{w}_{i}\right\|^{2}}{\left|\mathbf{w}_{i}^{H} \mathbf{H}^{H} \mathbf{e}_{i}\right|^{2}}, \quad i=1, \cdots, K .
$$

Let us multiply (85) with $\sigma_{i}^{2}$ for $i=1, \cdots, K$, and sum over $i$ on both sides:

$$
\left(1+\frac{1}{\gamma_{o}}\right) \sum_{i} \sigma_{i}^{2} \lambda_{i}=\sum_{i} \sigma_{i}^{2} \sum_{j} \frac{\left|\mathbf{w}_{i} \mathbf{H}^{H} \mathbf{e}_{j}\right|^{2}}{\left|\mathbf{w}_{i}^{H} \mathbf{H}^{H} \mathbf{e}_{i}\right|^{2}} \lambda_{j}+\sum_{i} \sigma_{i}^{2} \frac{\left\|\mathbf{H}_{\mathrm{Tx}} \mathbf{w}_{i}\right\|^{2}}{\left|\mathbf{w}_{i}^{H} \mathbf{H}^{H} \mathbf{e}_{i}\right|^{2}} .
$$

Similarly to the other constraints, it can be shown the power constraint is also active, i.e., $\sum_{i} \sigma_{i}^{2} \lambda_{i}=P_{o}$. Thus,

$$
\left(1+\frac{1}{\gamma_{o}}\right)=\frac{1}{P_{o}} \sum_{i} \sigma_{i}^{2} \sum_{j} \frac{\left|\mathbf{w}_{i} \mathbf{H}^{H} \mathbf{e}_{j}\right|^{2}}{\left|\mathbf{w}_{i}^{H} \mathbf{H}^{H} \mathbf{e}_{i}\right|^{2}} \lambda_{j}+\frac{1}{P_{o}} \sum_{i} \sigma_{i}^{2} \frac{\left\|\mathbf{H}_{\mathrm{Tx}} \mathbf{w}_{i}\right\|^{2}}{\left|\mathbf{w}_{i}^{H} \mathbf{H}^{H} \mathbf{e}_{i}\right|^{2}} .
$$


Rewriting the $K$ equalities of (85) and the equality of (87) in matrix form:

$$
\left(1+\frac{1}{\gamma_{o}}\right)\left[\begin{array}{c}
\boldsymbol{\lambda} \\
1
\end{array}\right]=\left[\begin{array}{cc}
\mathbf{Q} & \mathbf{q} \\
\frac{1}{P_{o}} \mathbf{v}^{T} \mathbf{Q} & \frac{1}{P_{o}} \mathbf{v}^{T} \mathbf{q}
\end{array}\right]\left[\begin{array}{c}
\boldsymbol{\lambda} \\
1
\end{array}\right]
$$

where $\boldsymbol{\lambda}$ is a length $K$ vector with elements $\lambda_{i}, \mathbf{Q}$ is a $K \times K$ matrix with elements $[\mathbf{Q}]_{i, j}=\frac{\left|\mathbf{w}_{i} \mathbf{H}^{H} \mathbf{e}_{j}\right|^{2}}{\left|\mathbf{w}_{i}^{H} \mathbf{H}^{H} \mathbf{e}_{i}\right|^{2}}, \mathbf{q}$ is a length $K$ vector with elements $\mathbf{q}_{i}=\frac{\left\|\mathbf{H}_{\mathrm{Tx}} \mathbf{w}_{i}\right\|^{2}}{\left|\mathbf{w}_{i}^{H} \mathbf{H}^{H} \mathbf{e}_{i}\right|^{2}}$, and $\mathbf{v}$ is a length $K$ vector with elements $\sigma_{i}^{2}$. It is easy to see that (88) defines an eigensystem. The optimal $\left(1+\frac{1}{\gamma_{0}}\right)$ is its maximal eigenvalue, and the optimal $\boldsymbol{\lambda}$ is the eigenvector associated with this maximal eigenvalue scaled so its last element will be one. For more details on this procedure (in the special case of $\mathbf{H}_{\mathrm{Tx}}=\mathbf{I}$ ) please refer to [13,48]. Clearly, this solution is much less appealing than the fixed point iteration in (80)-(81).

\section{SPECIAL CASES}

In this section, we examine a few interesting cases in which the problems $\mathcal{P}$ and $\mathcal{S}$ have simple closed forms.

\subsection{DIAGONAL CASE}

The first case is when the matrices $\mathbf{H}$ and $\mathbf{H}_{\mathrm{Tx}}$ are diagonal. In this case, it is easy to satisfy the optimality conditions in Theorem 1. The solution to the power optimization in (9) is

$$
\begin{aligned}
P_{o} & =\gamma_{o} \sum_{i} \frac{\sigma_{i}^{2}\left[\mathbf{H}_{\mathrm{Tx}}\right]_{i, i}^{2}}{[\mathbf{H}]_{i, i}^{2}} ; \\
{[\mathbf{T}]_{i, i} } & =\sqrt{\gamma_{o}} \operatorname{diag}\left\{\frac{\sigma_{i}}{[\mathbf{H}]_{i, i}}\right\} .
\end{aligned}
$$

The solution to the SIR optimization in (10) is

$$
\begin{aligned}
\gamma_{o} & =\frac{P_{o}}{\sum_{i} \frac{\sigma_{i}^{2}\left[\mathbf{H}_{\mathrm{Tx}}\right]_{i, i}^{2}}{[\mathbf{H}]_{i, i}^{2}}} ; \\
{[\mathbf{T}]_{i, i} } & =\sqrt{\frac{P_{o}}{\sum_{i} \frac{\sigma_{i}^{2}\left[\mathbf{H}_{\mathrm{Tx}}\right]_{i, i}^{2}}{[\mathbf{H}]_{i, i}^{2}}}} \operatorname{diag}\left\{\frac{\sigma_{i}}{[\mathbf{H}]_{i, i}}\right\} .
\end{aligned}
$$

The resulting precoders in these cases are simple power allocation strategies. 


\subsection{SYMMETRIC CASE}

The second case is when the matrices $\mathbf{H}$ and $\mathbf{H}_{\mathrm{Tx}}$ have equal diagonal elements and equal off diagonal elements, and the variances are equal $\sigma_{i}^{2}=\sigma^{2}$. Due to the symmetry it is clear that choosing $\lambda_{i}=P_{o} /\left(K \sigma^{2}\right)$ will satisfy the conditions in Theorem 1 . Therefore, the solution for the SIR optimization problem is:

$$
\begin{aligned}
& \gamma_{o}=\frac{1}{\left[\mathbf{H}\left(\mathbf{H}^{H} \mathbf{H}+\frac{K \sigma^{2}}{P_{O}} \mathbf{H}_{\mathrm{Tx}}^{H} \mathbf{H}_{\mathrm{Tx}}\right)^{-1} \mathbf{H}^{H}\right]_{i, i}}-1 \\
& \mathbf{T}=c\left[\mathbf{H}^{H} \mathbf{H}+\frac{K \sigma^{2}}{P_{o}} \mathbf{H}_{\mathrm{Tx}}^{H} \mathbf{H}_{\mathrm{Tx}}\right]^{\dagger} \mathbf{H}^{H},
\end{aligned}
$$

where $c$ is a constant that scales the matrix to satisfy the power constraint. This particular precoder has been previously derived in $[7,8]$ and recently in $[9,10]$ through scaled MMSE considerations. However, it is easy to verify that in general, i.e., in nonsymmetric channels, it does not necessarily satisfy the conditions in Theorem 1, and is therefore suboptimal in this sense. For example, if the channels are symmetric but the noise variances are not equal, then, in order to ensure equal SINRs among all the streams, the precoder in (94) must be diagonally scaled using (42).

\section{APPLICATIONS IN MULTIPLE USER SYSTEMS}

In this section, we present possible applications of the proposed precoders. Consider a multiple user precoded downlink system. At each symbol's period the base station transmits using an $N \times K$ non orthogonal signatures matrix $\mathbf{H}_{\mathrm{Tx}}=\mathbf{S}$. The cross correlations between the signatures are denoted by $\rho_{i, j}=\left[\mathbf{S}^{H} \mathbf{S}\right]_{i, j}$. For simplicity, we assume ideal channels, i.e., $\mathbf{H}_{\mathrm{Ch}, i}=\mathbf{I}$. Denoting by $\mathbf{y}$ the output vector of the multiple user receiver, we have that

$$
\mathbf{y}=\mathbf{H}_{\mathrm{Rx}} \mathbf{S T b}+\mathbf{H}_{\mathrm{Rx}} \mathbf{w}
$$

where $\mathbf{H}_{\mathrm{Rx}}$ is one of the standard filters:

- MF receiver: $\mathbf{H}_{\mathrm{Rx}}=\mathbf{S}^{H}$.

- ZF receiver: $\mathbf{H}_{\mathrm{Rx}}=\left(\mathbf{S}^{H} \mathbf{S}\right)^{-1} \mathbf{S}^{H}$.

- MMSE receiver: $\mathbf{H}_{\mathrm{Rx}}=\mathbf{S}^{H}\left(\mathbf{S S}^{H}+\operatorname{diag}\left\{\sigma_{i}^{2}\right\}\right)^{-1}$.

We now discuss the performance of these systems with and without precoding. 


\subsection{EQUAL POWER AND EQUAL CROSS CORRELATIONS}

The first interesting result of our precoder is its performance in an equal power and equal cross correlations multiuser system, i.e. $\rho_{i, j}=\rho$ and $\sigma_{i}^{2}=\sigma^{2}$. As explained in Section 6.2, our precoder and its SINRs have closed forms in this case.

Lemma 2. Consider the multiuser system in (95). If $\mathbf{S}^{H} \mathbf{S}$ is invertible, $\rho_{i, j}=\rho$ and $\sigma_{i}^{2}=\sigma^{2}$, then the output SINRs using the $\mathcal{S}$ precoder along with an $M F$ receiver are identical to those resulting by using an MMSE receiver without any precoder, and are equal to

$$
\gamma_{o}=\frac{1}{\left[\left(\mathbf{I}+\frac{P_{o}}{K \sigma^{2}} \mathbf{S}^{H} \mathbf{S}\right)^{-1}\right]_{i, i}}-1
$$

Proof. Using (93) and the matrix inversion lemma, the output SINRs can be derived as

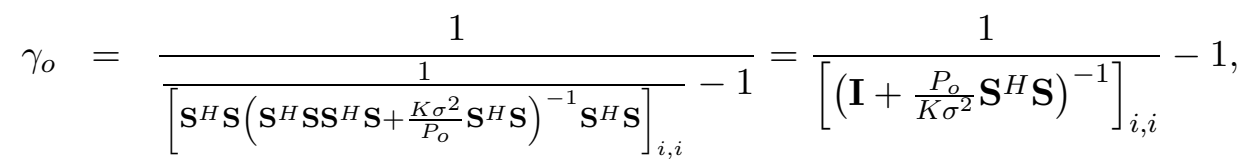

In [3], it is shown that the output SINRs using MMSE receivers are also equivalent to (96).

This result is interesting, as it allows for each user to attain the MMSE performance without the use of an MMSE receiver which requires the knowledge of all the other signatures and a matrix inversion. Moreover, when the $\mathcal{S}$ precoder is used with ZF or MMSE receivers the performance improves even more. In Fig. 4 we plot the output SINRs given by (93) for the three linear receivers. For comparison, we also plot the output SINRs that result from similar systems without a precoder [3]. As expected, using the precoder always improves the output SINR.

\subsection{NON SYMMETRIC CHANNEL}

As a second example, we consider an equal power system with unequal cross correlations between the users signatures. In such systems, there is no closed form expression for the performance. Therefore, we resort to Monte Carlo simulations. Following [5], we simulate the cross correlations $\rho_{12}=0.8, \rho_{13}=0.9$, and $\rho_{23}=0.7$. Each user uses an MF receiver. For comparison, we provide results of the decorrelator precoder [5], and our SIR precoder of (10). Due to the asymmetry, each of the three users performs differently when using the previous precoders. On the other hand, our precoder has the attractive property of equal BERs for all the users. Naturally, the performance of the best user degrades compared to previous methods. In Fig. 5, 


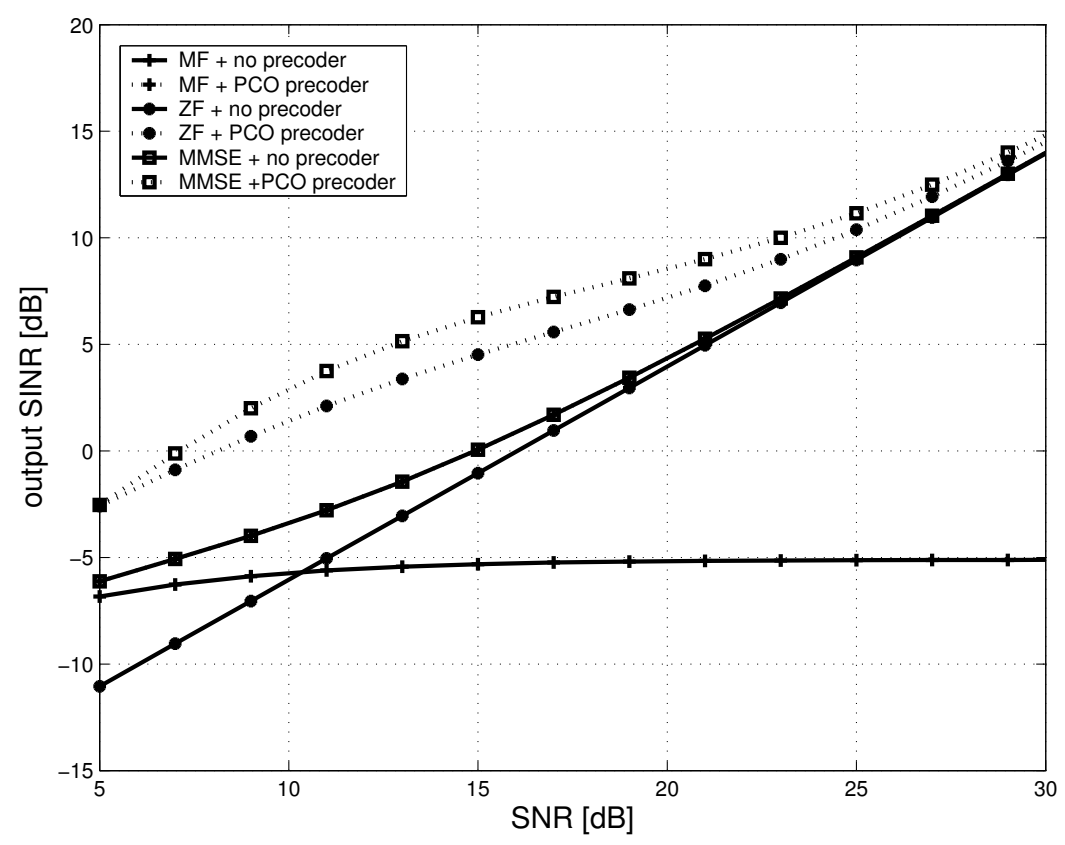

Figure 4: SINR of a symmetric system with equal cross correlations. Due to the symmetry, all users have equal output SINRs.

we provide the BERs using each of the precoders. It is easy to see that the our precoder outperforms that of $[5]$.

\subsection{RANK DEFICIENT CHANNELS}

As explained in the introduction, one of the main advantages of our precoder over previous ones is its performance in rank deficient systems. We now illustrate this property in a multiuser system with $K=4$ users and length $N=3$ sequences. The transmitter uses the optimal sequences of [37], and the receiver uses conventional matched filters. However, we use a distorting channel for the first user, i.e., $\mathbf{H}_{\mathrm{Ch}, 1}$ is a toeplitz matrix with the first row $[1.0,0.8,0.0]$. Due to this channel the sequences are no longer optimal and a precoder should be used. The common decorrelating precoder of [5] cannot be derived in this case as $N<K$. Therefore, we compare our results to the precoder of [7]. The worst output SINRs with and without the precoders are presented in Fig. 6. Using our $\mathcal{S}$ precoder significantly increases the SINR compared to a system with no precoder. Using the precoder, the SINRs asymptotically converge to the bound in (19), i.e. $\gamma_{i}=\frac{1}{\frac{4}{3}-1}=3$ for $i=1, \cdots, 4$. Interestingly, the performance using the precoder of [7] is even worse than not using a precoder at all. For fairness, we must note that the SINR of the best user using this precoder are much higher. But from a system's prespective, the interesting metric is the performance of the worst user, and therefore our precoder is more appealing. 


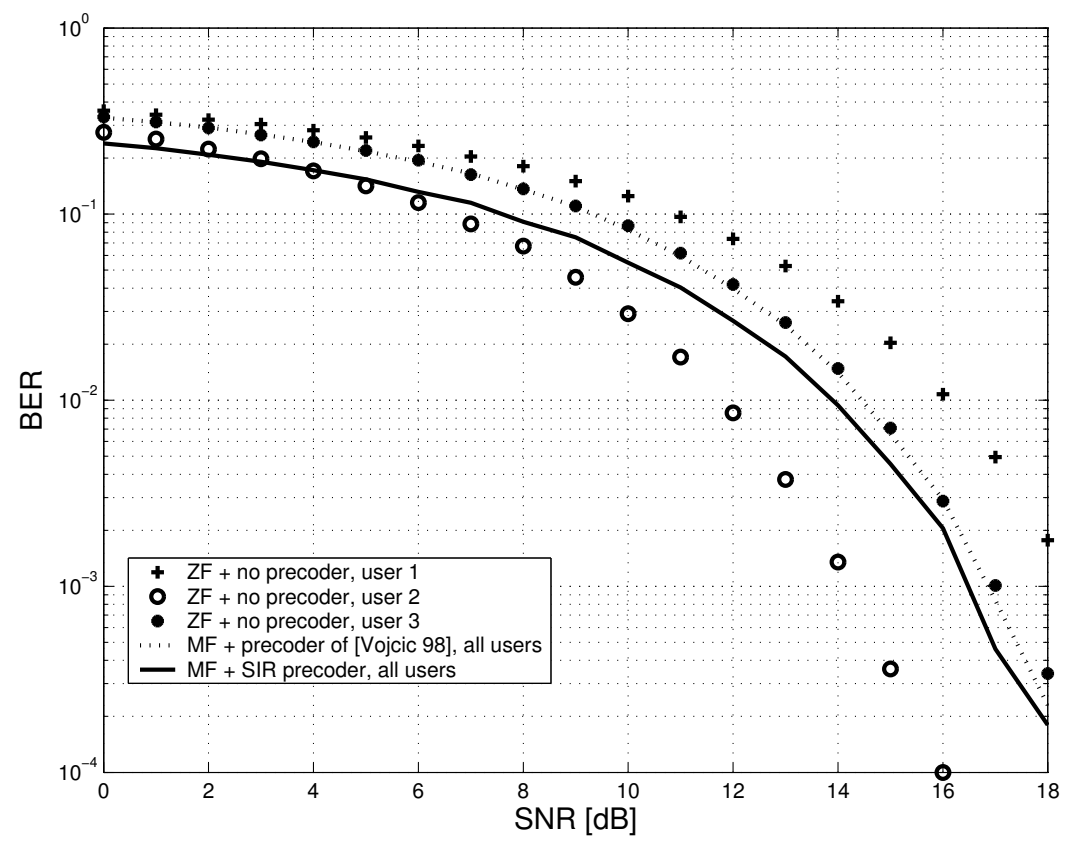

Figure 5: SINR of a non symmetric 3 users system with cross correlations $\rho_{12}=0.8, \rho_{13}=0.9, \rho_{23}=0.7$.

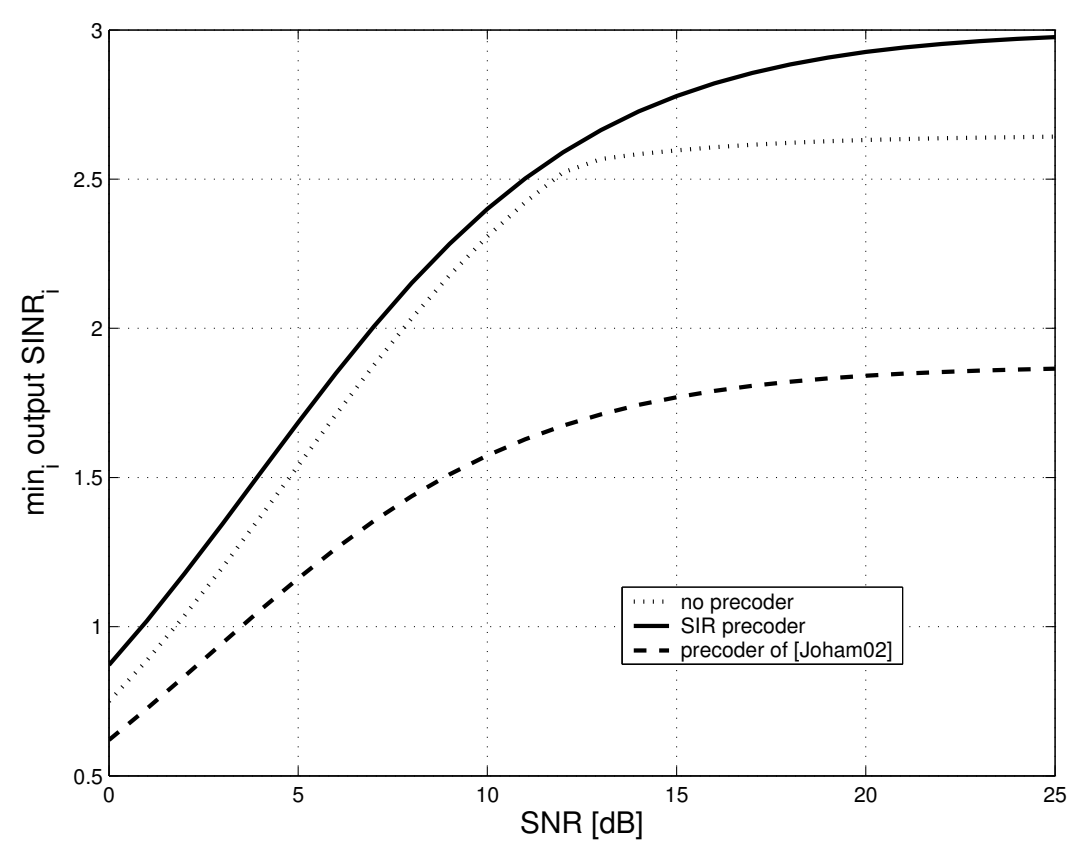

Figure 6: Worst output SINR in a system with $K=4$ and $N=3$. 


\section{CONCLUSION AND FUTURE WORK}

In this paper, we addressed the problem of designing linear precoders for fixed MIMO receivers. We considered two complementary design criteria, and proposed numerous alternative algorithmic solutions for these optimization problems.

It is first observed that in precoder design, maximizing the worst SINR is advantageous to minimizing MSE. Most of the previous work regarding precoders is based on optimizing variants of the MMSE criterion. These ad hoc criteria are usually computationally attractive and perform quite well. However, the ever increasing demand for better performance, as well as the considerable progress in optimization theory, suggests that upcoming research should focus on design criteria which are more related to practical performance measures, such as maximizing the worst SINR.

Our second important observation is that by using conic optimization theory and algorithms, the precoder design problems can be solved in a straight forward manner without using downlink uplink duality. This duality is remarkable, and has enabled solutions to problems which were unsolvable before. Nonetheless, we believe that understanding the precoder design on first principles, and not as a byproduct of the uplink problem, is also important. For example, in future work, the simple optimality conditions may help in analyzing the performance of these systems, or in improving the design criteria, without the need to always return to the virtual uplink problem.

There are many interesting extensions to this work which are worth pursuing. The first concerns the extension of our results to the case of partial CSI. In many practical systems, the transmitter does not have access to perfect CSI, and needs to resort to noisy channel estimates, and/or delayed feedback. In this case, robust optimization algorithms should be applied as in [59]. Another possible direction is to consider fixed non linear receivers, such as the successive cancelling receiver. It is well known that such receivers outperform the linear receivers explored in our paper. Therefore, by designing the precoder to optimally work with such receivers, the performance can significantly improve.

\section{A OPTIMALITY CONDITIONS FOR PROGRAMS WITH SOC CON- STRAINTS}

In this appendix, we derive optimality conditions for optimization programs with SOC constraints. The conditions are summarized in the following lemma. 
Lemma 3. Consider a non convex program of the structure

$$
\begin{cases}\min _{\mathbf{X}} & f(\mathbf{X}) \\ \text { s.t. } & \left\|\mathbf{a}_{i}(\mathbf{X})\right\|^{2}-\left|a_{i}(\mathbf{X})\right|^{2} \leq 0, \quad i=1, \cdots, N .\end{cases}
$$

where $f(\mathbf{X})$ is convex in $\mathbf{X}$, and $a_{i}(\mathbf{X})$ and $\mathbf{a}_{i}(\mathbf{X})$ for $=1, \cdots, K$, are affine functions of $\mathbf{X}$. Let us associate the dual variables $\lambda_{i}$ for $i=1, \cdots, K$ with this program. If the program is strictly feasible, then the following KKT conditions are necessary and sufficient conditions for optimality of $\mathbf{X}$ and $\lambda_{i}$ :

1. Feasibility - the variable $\mathbf{X}$ is feasible

$$
\left|a_{i}(\mathbf{X})\right|^{2} \geq\left\|\mathbf{a}_{i}(\mathbf{X})\right\|^{2}, \quad i=1, \cdots, K,
$$

and the dual variables are dual feasible $\lambda_{i} \geq 0$ for $i=1, \cdots, K$.

2. Complementary Slackness - for each $i=1, \cdots, K$, one of the following conditions hold

$$
\lambda_{i}=0 \quad \text { or } \quad\left|a_{i}(\mathbf{X})\right|^{2}=\left\|\mathbf{a}_{i}(\mathbf{X})\right\|^{2} .
$$

3. Zero derivative - the derivative of the the Lagrangian of (98) with respect to $\mathbf{X}$ is zero

$$
\frac{\partial}{\partial \mathbf{X}}\left\{f(\mathbf{X})+\sum_{i} \lambda_{i}\left[\left\|\mathbf{a}_{i}(\mathbf{X})\right\|^{2}-\left|a_{i}(\mathbf{X})\right|^{2}\right]\right\}=\mathbf{0}
$$

Proof. For simplicity, we will only deal with real valued variables and functions. The extension to complex values is straight forward. The KKT conditions are necessary for optimality of any optimization problem [18]. If the program in (98) was in convex form, then the conditions were also sufficient for optimality. Unfortunately, the program is not expressed in convex form and therefore we must prove the sufficiency. Let us begin by rewriting (98) in convex form:

$$
\begin{cases}\min _{\mathbf{X}} & f(\mathbf{X}) \\
\text { s.t. } & {\left[\begin{array}{l}
a_{i}(\mathbf{X}) \\
\mathbf{a}_{i}(\mathbf{X})
\end{array}\right] \succeq_{K} 0, \quad i=1, \cdots, N .}\end{cases}
$$

If (102) is strictly feasible, then the following conic KKT conditions are necessary and sufficient for optimality [18]: 
1. Feasibility: The primal variable $\mathbf{X}$ is feasible, and the associated dual cones are dual feasible:

$$
\left[\begin{array}{c}
a_{i}(\mathbf{X}) \\
\mathbf{a}_{i}(\mathbf{X})
\end{array}\right] \succeq_{K}, \quad\left[\begin{array}{c}
w_{i} \\
\mathbf{w}_{i}
\end{array}\right] \succeq_{K} 0, \quad i=1, \cdots, K .
$$

2. Complementary Slackness:

$$
\left[\begin{array}{cc}
w_{i} & \mathbf{w}_{i}^{T}
\end{array}\right]\left[\begin{array}{c}
a_{i}(\mathbf{X}) \\
\mathbf{a}_{i}(\mathbf{X})
\end{array}\right]=0, \quad i=1, \cdots, K
$$

3. Zero derivative: The derivative of the Lagrangian ${ }^{3}$ of (102) with respect to $\mathbf{X}$ is zero

$$
\frac{\partial}{\partial \mathbf{X}}\left\{f(\mathbf{X})-\sum_{i}\left(\left[\begin{array}{ll}
w_{i} & \mathbf{w}_{i}^{T}
\end{array}\right]\left[\begin{array}{c}
a_{i}(\mathbf{X}) \\
\mathbf{a}_{i}(\mathbf{X})
\end{array}\right]\right)\right\}=\mathbf{0} .
$$

We now show that the conditions in (99)-(101) are sufficient for satisfying the conditions in (103)-(105). Let us choose:

$$
\left[\begin{array}{c}
w_{i} \\
\mathbf{w}_{i}
\end{array}\right]=2 \lambda_{i}\left[\begin{array}{c}
a_{i}(\mathbf{X}) \\
-\mathbf{a}_{i}(\mathbf{X})
\end{array}\right], \quad i=1, \cdots, K
$$

Plugging the dual variables from (106) into the conic KKT conditions reveals that conditions (103)-(104) hold due to (99)-(100). Similarly, using (106), the conditions in (101) and (105) are identical:

$$
\begin{aligned}
\frac{\partial}{\partial \mathbf{X}}\left\{f(\mathbf{X})-\sum_{i}\left(\left[\begin{array}{ll}
w_{i} & \mathbf{w}_{i}^{T}
\end{array}\right]\left[\begin{array}{c}
a_{i}(\mathbf{X}) \\
\mathbf{a}_{i}(\mathbf{X})
\end{array}\right]\right)\right\} & =\frac{\partial f(\mathbf{X})}{\partial \mathbf{X}}-\sum_{i}\left(2 \lambda_{i} a_{i}(\mathbf{X}) \frac{\partial a_{i}(\mathbf{X})}{\partial \mathbf{X}}-2 \lambda_{i} \mathbf{a}_{i}^{T}(\mathbf{X}) \frac{\partial \mathbf{a}_{i}(\mathbf{X})}{\partial \mathbf{X}}\right) \\
& =\frac{\partial}{\partial \mathbf{X}}\left\{f(\mathbf{X})+\sum_{i} \lambda_{i}\left(\left\|\mathbf{a}_{i}(\mathbf{X})\right\|^{2}-\left|a_{i}(\mathbf{X})\right|^{2}\right)\right\}
\end{aligned}
$$

\footnotetext{
${ }^{3}$ The Lagrangian is formulated by subtracting the product of the dual cones with the primal cones. The products are subtracted instead of added (as in regular convex programming) because the SOC is defined as a "greater than or equal" generalized inequality and not as a "less than or equal" generalized inequality [18].
} 


\section{B PROPERTIES OF $f_{i}(\boldsymbol{\Lambda})$}

Consider the functions:

$$
f_{i}(\boldsymbol{\Lambda})=\frac{\gamma_{o}}{1+\gamma_{o}} \frac{1}{\left[\mathbf{H}\left(\mathbf{H}^{H} \boldsymbol{\Lambda} \mathbf{H}+\mathbf{H}_{\mathrm{Tx}}^{H} \mathbf{H}_{\mathrm{Tx}}\right)^{\dagger} \mathbf{H}^{H}\right]_{i, i}}, \quad i=1, \cdots, K,
$$

In this appendix, we will prove some properties of $f_{i}(\boldsymbol{\Lambda})$. The proofs rely on the following lemma:

Lemma 4. If $\mathbf{A} \succeq 0, \mathbf{B} \succeq 0$ and $\mathbf{c}$ is in the range of $\mathbf{A}$, then

$$
\frac{1}{\mathbf{c}^{H}(\mathbf{A}+\mathbf{B})^{\dagger} \mathbf{c}} \geq \frac{1}{\mathbf{c}^{H} \mathbf{A}^{\dagger} \mathbf{c}}
$$

with equality if and only if $\mathbf{B}(\mathbf{A}+\mathbf{B})^{\dagger} \mathbf{c}=\mathbf{0}$.

Proof. First note that if $\mathbf{c}$ is in the range of $\mathbf{A}$ then

$$
\mathcal{Q}(\mathbf{A}, \mathbf{c})=\frac{1}{\mathbf{c}^{H} \mathbf{A}^{\dagger} \mathbf{c}}=\left\{\begin{array}{cc}
\min _{\mathbf{x}} & \mathbf{x}^{H} \mathbf{A} \mathbf{x} \\
\text { s.t. } & \mathbf{c}^{H} \mathbf{x}=1
\end{array}\right.
$$

As proof, let us derive the Lagrangian of (110):

$$
\mathcal{L}=\mathbf{x}^{H} \mathbf{A} \mathbf{x}+\lambda\left(\mathbf{c}^{H} \mathbf{x}-1\right)
$$

Equating the derivative with respect to $\mathbf{x}$ to zero yields

$$
\frac{\partial \mathcal{L}}{\partial \mathbf{x}}=2 \mathbf{A x}+\lambda \mathbf{c}=\mathbf{0}
$$

Clearly, the solution to this condition is $\mathbf{x}=\frac{\mathbf{A}^{\dagger} \mathbf{c}}{\mathbf{c}^{H} \mathbf{A}^{\dagger} \mathbf{c}}+\mathbf{y}$ and $\lambda=-2 \frac{1}{\mathbf{c}^{H} \mathbf{A}^{\dagger} \mathbf{c}}$, where $\mathbf{y}$ is any vector in the null space of $\mathbf{A}$.

Using (110), we need to prove that

$$
\mathcal{Q}(\mathbf{A}+\mathbf{B}, \mathbf{c}) \geq \mathcal{Q}(\mathbf{A}, \mathbf{c}) .
$$

Let us denote the optimal argument of $\mathcal{Q}(\mathbf{A}, \mathbf{c})$ by $\mathbf{x}_{A}$ and the optimal argument of $\mathcal{Q}(\mathbf{A}+\mathbf{B}, \mathbf{c})$ by $\mathbf{x}_{A+B}$. In order to prove the inequality, assume the contrary, i.e., the optimal value of $\mathcal{Q}(\mathbf{A}+\mathbf{B}, \mathbf{c})$ is less than that of $\mathcal{Q}(\mathbf{A}, \mathbf{c})$. Then, this is a contradiction to the optimality of $\mathbf{x}_{A}$, because $\mathbf{x}_{A+B}$ is feasible for $\mathcal{Q}(\mathbf{A}, \mathbf{c})$ 
and results in a smaller objective value.

In order to prove the case of strict inequality, we examine the case when

$$
\mathcal{Q}(\mathbf{A}+\mathbf{B}, \mathbf{c})=\mathcal{Q}(\mathbf{A}, \mathbf{c}) .
$$

Due to the optimality of $\mathbf{x}_{A}$, we have $\mathbf{x}_{A}^{H} \mathbf{A} \mathbf{x}_{A} \leq \mathbf{x}_{A+B}^{H} \mathbf{A} \mathbf{x}_{A+B}$. But, due to (114), we have $\mathbf{x}_{A}^{H} \mathbf{A} \mathbf{x}_{A}=$ $\mathbf{x}_{A+B}^{H} \mathbf{A} \mathbf{x}_{A+B}+\mathbf{x}_{A+B}^{H} \mathbf{B} \mathbf{x}_{A+B}$. These conditions hold together if and only if $\mathbf{x}_{A+B}^{H} \mathbf{B} \mathbf{x}_{A+B}=0$. Plugging in the optimal $\mathbf{x}_{A+B}$ yields $\mathbf{c}^{H}(\mathbf{A}+\mathbf{B})^{\dagger} \mathbf{B}(\mathbf{A}+\mathbf{B})^{\dagger} \mathbf{c}=0$. Finally, due to semidefiniteness of $\mathbf{B}$, this is possible only if $\mathbf{B}(\mathbf{A}+\mathbf{B})^{\dagger} \mathbf{c}=\mathbf{0}$.

Using Lemma 4 with $\mathbf{c}=\mathbf{H}^{H} \mathbf{e}_{i}$ (which is in the range of $\mathbf{H}_{\mathrm{Tx}}^{H} \mathbf{H}_{\mathrm{Tx}}$ ), we prove the following properties:

- Positivity - if $\lambda_{i} \geq 0$ for $i=1, \cdots, K$, then $f_{j}(\boldsymbol{\Lambda})>0$ for $j=1, \cdots, K$.

Proof. Observe the following chain:

$$
\begin{aligned}
f_{i}(\boldsymbol{\Lambda}) & =\frac{\gamma_{o}}{1+\gamma_{o}} \frac{1}{\left[\mathbf{H}\left(\mathbf{H}^{H} \boldsymbol{\Lambda} \mathbf{H}+\mathbf{H}_{\mathrm{Tx}}^{H} \mathbf{H}_{\mathrm{Tx}}\right)^{\dagger} \mathbf{H}^{H}\right]_{i, i}} \\
& \geq \frac{\gamma_{o}}{1+\gamma_{o}} \frac{1}{\left[\mathbf{H}\left(\mathbf{H}_{\mathrm{Tx}}^{H} \mathbf{H}_{\mathrm{Tx}}\right)^{\dagger} \mathbf{H}^{H}\right]_{i, i}}, \quad i=1, \cdots, K,
\end{aligned}
$$

where we have used Lemma 4 with $\mathbf{A}=\mathbf{H}_{\mathrm{Tx}}^{H} \mathbf{H}_{\mathrm{Tx}}$ and $\mathbf{B}=\mathbf{H}^{H} \boldsymbol{\Lambda} \mathbf{H}$. Due to the semi definiteness, the diagonal elements of $\mathbf{H}\left(\mathbf{H}_{\mathrm{Tx}}^{H} \mathbf{H}_{\mathrm{Tx}}\right)^{\dagger} \mathbf{H}^{H}$ are non negative. Therefore, if they are finite, then their inverses are positive, and the property holds.

- Monotonicity - if $\lambda_{i} \geq \lambda_{i}^{\prime}$ for $i=1, \cdots, K$, then $f_{j}(\boldsymbol{\Lambda}) \geq f_{j}\left(\boldsymbol{\Lambda}^{\prime}\right)$ for $j=1, \cdots, K$.

Proof. Observe the following chain:

$$
\begin{aligned}
f_{i}(\boldsymbol{\Lambda}) & =\frac{\gamma_{o}}{1+\gamma_{o}} \frac{1}{\left[\mathbf{H}\left(\mathbf{H}^{H} \boldsymbol{\Lambda} \mathbf{H}+\mathbf{H}_{\mathrm{Tx}}^{H} \mathbf{H}_{\mathrm{Tx}}\right)^{\dagger} \mathbf{H}^{H}\right]_{i, i}} \\
& =\frac{\gamma_{o}}{1+\gamma_{o}} \frac{1}{\left[\mathbf{H}\left(\mathbf{H}^{H} \operatorname{diag}\left\{\lambda_{i}^{\prime}\right\} \mathbf{H}+\mathbf{H}_{\mathrm{Tx}}^{H} \mathbf{H}_{\mathrm{Tx}}+\mathbf{H}^{H} \operatorname{diag}\left\{\lambda_{i}-\lambda_{i}^{\prime}\right\} \mathbf{H}^{\dagger} \mathbf{H}^{H}\right]_{i, i}\right.} \\
& \geq \frac{\gamma_{o}}{1+\gamma_{o}} \frac{1}{\left[\mathbf{H}\left(\mathbf{H}^{H} \operatorname{diag}\left\{\lambda_{i}^{\prime}\right\} \mathbf{H}+\mathbf{H}_{\mathrm{Tx}}^{H} \mathbf{H}_{\mathrm{Tx}}\right)^{\dagger} \mathbf{H}^{H}\right]_{i, i}} \\
& =f_{i}\left(\boldsymbol{\Lambda}^{\prime}\right), \quad i=1, \cdots, K,
\end{aligned}
$$

where we have used Lemma 4 with $\mathbf{A}=\mathbf{H}^{H} \operatorname{diag}\left\{\lambda_{i}^{\prime}\right\} \mathbf{H}+\mathbf{H}_{\mathrm{Tx}}^{H} \mathbf{H}_{\mathrm{Tx}}$ and $\mathbf{B}=\mathbf{H}^{H} \operatorname{diag}\left\{\lambda_{i}-\lambda_{i}^{\prime}\right\} \mathbf{H}$ 
- Scalability - if $\alpha>1$, then $\alpha f_{i}(\boldsymbol{\Lambda})>f_{i}(\alpha \boldsymbol{\Lambda})$ for $j=1, \cdots, K$.

Proof. Observe the following chain:

$$
\begin{aligned}
\alpha f_{i}(\boldsymbol{\Lambda}) & =\alpha \frac{\gamma_{o}}{1+\gamma_{o}} \frac{1}{\left[\mathbf{H}\left(\mathbf{H}^{H} \boldsymbol{\Lambda} \mathbf{H}+\mathbf{H}_{\mathrm{Tx}}^{H} \mathbf{H}_{\mathrm{Tx}}\right)^{\dagger} \mathbf{H}^{H}\right]_{i, i}} \\
& =\frac{\gamma_{o}}{1+\gamma_{o}} \frac{1}{\left[\mathbf{H}\left(\mathbf{H}^{H} \operatorname{diag}\left\{\alpha \lambda_{i}\right\} \mathbf{H}+\mathbf{H}_{\mathrm{Tx}}^{H} \mathbf{H}_{\mathrm{Tx}}+(\alpha-1) \mathbf{H}_{\mathrm{Tx}}^{H} \mathbf{H}_{\mathrm{Tx}}\right)^{\dagger} \mathbf{H}^{H}\right]_{i, i}} \\
& \geq \frac{1}{1+\gamma_{o}} \frac{1}{\left[\mathbf{H}\left(\mathbf{H}^{H} \operatorname{diag}\left\{\alpha \lambda_{i}\right\} \mathbf{H}+\mathbf{H}_{\mathrm{Tx}}^{H} \mathbf{H}_{\mathrm{Tx}}\right)^{\dagger} \mathbf{H}^{H}\right]_{i, i}} \\
& =f_{i}(\alpha \boldsymbol{\Lambda}), \quad i=1, \cdots, K,
\end{aligned}
$$

where we have used Lemma 4 with $\mathbf{A}=\mathbf{H}^{H} \operatorname{diag}\left\{\alpha \lambda_{i}\right\} \mathbf{H}+\mathbf{H}_{\mathrm{Tx}}^{H} \mathbf{H}_{\mathrm{Tx}}$ and $\mathbf{B}=(\alpha-1) \mathbf{H}_{\mathrm{Tx}}^{H} \mathbf{H}_{\mathrm{Tx}}$. The inequality is non strict, i.e., holds with equality if and only if $\mathbf{H}_{\mathrm{Tx}}(\mathbf{A}+\mathbf{B})^{\dagger} \mathbf{H}^{H} \mathbf{e}_{i}=\mathbf{0}$. Multiplying by $\mathbf{e}_{i}^{H} \mathbf{H}_{\mathrm{RxCh}}$ on the left, yields $\mathbf{e}_{i}^{H} \mathbf{H}(\mathbf{A}+\mathbf{B})^{\dagger} \mathbf{H}^{H} \mathbf{e}_{i}=\mathbf{0}$. Therefore $\mathbf{H}^{H} \mathbf{e}_{i} \in \mathcal{N}\left\{(\mathbf{A}+\mathbf{B})^{\dagger}\right\}$, and due to the symmetry of $\mathbf{A}+\mathbf{B}$, we also have $\mathbf{H}^{H} \mathbf{e}_{i} \in \mathcal{N}\{\mathbf{A}+\mathbf{B}\}$. In addition, due to the semi definiteness, this means that $\mathbf{H}^{H} \mathbf{e}_{i} \in \mathcal{N}\left\{\mathbf{H}^{H} \operatorname{diag}\left\{\alpha \lambda_{i}\right\} \mathbf{H}\right\}$ and $\mathbf{H}^{H} \mathbf{e}_{i} \in \mathcal{N}\left\{\mathbf{H}_{\mathrm{Tx}}^{H} \mathbf{H}_{\mathrm{Tx}}\right\}$. Therefore, $\mathbf{H}_{\mathrm{Tx}}^{H} \mathbf{H}_{\mathrm{Tx}} \mathbf{H}_{\mathrm{Tx}}^{H} \mathbf{H}_{\mathrm{ChRx}}^{H} \mathbf{e}_{i}=\mathbf{0}$, and $\mathbf{H}_{\mathrm{Tx}}^{H} \mathbf{H}_{\mathrm{ChRx}}^{H} \mathbf{e}_{i}=\mathbf{0}$. Consequently, the problem is infeasible.

\section{DERIVATION OF DUAL PROGRAM}

In this appendix, we derive the dual program (64) and prove that its optimal solution satisfies the conditions of Theorem 1. Let us rewrite the power optimization problem using simple scalar inequalities:

$$
\begin{cases}\min _{\mathbf{T}} & \operatorname{Tr}\left\{\mathbf{T}^{H} \mathbf{H}_{\mathrm{Tx}}^{H} \mathbf{H}_{\mathrm{Tx}} \mathbf{T}\right\} \\ \text { s.t. } & \sum_{j}\left|[\mathbf{H T}]_{i, j}\right|^{2}+\sigma_{i}^{2}-\left(1+\frac{1}{\gamma_{o}}\right)\left|[\mathbf{H T}]_{i, i}\right|^{2} \leq 0, \quad i=1, \cdots, K\end{cases}
$$

The above program is not in convex form, since its constraints are not defined as $f(\cdot) \leq 0$ where $f(\cdot)$ is convex in its arguments. Nonetheless, it will still have a concave dual program, whose optimal solution will lower bound its optimal objective value. Define the Lagrangian of (118):

$$
\begin{aligned}
\mathcal{L}\left(\mathbf{t}_{i}, \lambda_{i}\right) & =\operatorname{Tr}\left\{\mathbf{T}^{H} \mathbf{H}_{\mathrm{Tx}}^{H} \mathbf{H}_{\mathrm{Tx}} \mathbf{T}\right\}+\sum_{i} \lambda_{i}\left[\sum_{j}\left|[\mathbf{H} \mathbf{T}]_{i, j}\right|^{2}+\sigma_{i}^{2}-\left(1+\frac{1}{\gamma_{o}}\right)\left|[\mathbf{H} \mathbf{T}]_{i, i}\right|^{2}\right] \\
& =\sum_{i} \lambda_{i} \sigma_{i}^{2}+\sum_{i} \mathbf{t}_{i}^{H}\left[\mathbf{H}^{H} \boldsymbol{\Lambda} \mathbf{H}+\mathbf{H}_{\mathrm{Tx}}^{H} \mathbf{H}_{\mathrm{Tx}}-\left(1+\frac{1}{\gamma_{o}}\right) \lambda_{i} \mathbf{H}^{H} \mathbf{e}_{i} \mathbf{e}_{i}^{H} \mathbf{H}\right] \mathbf{t}_{i}
\end{aligned}
$$


where $\mathbf{t}_{i}=\mathbf{T e}_{i}$ for $i=1 \cdots K$. The dual function associated with (118) is obtained by minimizing $\mathcal{L}\left(\mathbf{t}_{i}, \boldsymbol{\Lambda}\right)$ over the primal variables $\mathbf{t}_{i}$ :

$$
\begin{aligned}
d\left(\lambda_{i}\right) & =\inf _{\mathbf{t}_{i}} \mathcal{L}\left(\mathbf{t}_{i}, \lambda_{i}\right) \\
& = \begin{cases}\sum_{i} \lambda_{i} \sigma_{i}^{2} & \mathbf{H}^{H} \boldsymbol{\Lambda} \mathbf{H}+\mathbf{H}_{\mathrm{Tx}}^{H} \mathbf{H}_{\mathrm{Tx}}-\left(1+\frac{1}{\gamma_{o}}\right) \lambda_{i} \mathbf{H}^{H} \mathbf{e}_{i} \mathbf{e}_{i}^{H} \mathbf{H} \succeq 0, \quad, i=1, \cdots, K ; \\
-\infty & \text { else. }\end{cases}
\end{aligned}
$$

where we have used the fact that the infimum of a quadratic form is either zero (if the form is positive semi definite) or $-\infty$ (if the form is not positive semi definite). Using the dual function, the dual program is defined as:

$$
\begin{array}{ll}
\max _{\lambda_{i}} & d\left(\lambda_{i}\right) \\
\text { s.t. } & \lambda_{i} \geq 0, \quad i=1, \cdots, K .
\end{array}
$$

Thus, the dual program associated with the power optimization is:

$$
\begin{cases}\max _{\lambda_{i} \geq 0} & \sum_{i} \lambda_{i} \sigma_{i}^{2} \\ \text { s.t. } & \frac{\gamma_{o}}{1+\gamma_{o}}\left[\mathbf{H}^{H} \boldsymbol{\Lambda} \mathbf{H}+\mathbf{H}_{\mathrm{Tx}}^{H} \mathbf{H}_{\mathrm{Tx}}\right]-\lambda_{i} \mathbf{H}^{H} \mathbf{e}_{i} \mathbf{e}_{i}^{H} \mathbf{H} \succeq 0, \quad i=1, \cdots, K .\end{cases}
$$

In general, there is no guaranty for zero duality gap between (118) and (122). Nor is there a guaranty that the solution of (122) will satisfy the conditions in Theorem 1. However, using the properties in Appendix B, we can prove that in this specific case, the conditions will be satisfied. A similar proof is available in [42], and is therefore omitted.

\section{References}

[1] A. Wiesel, Y. C. Eldar, and S. Shamai (Shitz), "Multiuser precoders for fixed receivers," in Proc. of IEEE Int. Zurich Seminar (IZS-2004), Feb. 2004.

[2] A. Wiesel, Y. C. Eldar, and S. Shamai (Shitz), "Linear precoder for fixed MIMO receivers," in Proc. of IEEE Int. Conf. on Acoustics, Speech and Signal Processing (ICASSP-2004), May 2004.

[3] S. Verdu, Multiuser Detection, Cambridge University Press, 1998.

[4] A. Goldsmith, S. A. Jafar, N. Jindal, and S. Vishwanath, "Capacity limits of MIMO channels," IEEE Journal on selected areas in Communications, vol. 21, pp. 684-702, June 2003. 
[5] B. R. Vojcic and W. M. Jang, "Transmitter precoding in synchronous multiuser commmunication," IEEE Trans. on Communications, vol. 46, no. 10, pp. 1346-1355, Oct. 1998.

[6] R. F.H. Fischer, Precoding and Signal Shaping for Digital Transmission, John Wiley and Sons, Inc., 2002.

[7] M. Joham, K. Kusume, M. H. Gzara, and W. Utschick, "Transmit wiener filter for the downlink of TDD DS-CDMA systems," in Proc. of IEEE 7th ISSSTA, Sept 2002.

[8] F. Wathan, R. Irmer, and G. Fettweis, "On transmitter-based interference mitigation in TDD-downlink with frequency selective fading environment," in Proc. of Asia-Pacific Conf. on Communications (APCC), Sept. 2002.

[9] R. L. U. Choi and R.D. Murch, "New transmit schemes and simplified receivers for MIMO wireless communications systems," IEEE Trans. on Wireless Communications, vol. 2, no. 6, pp. 1217-1230, Nov. 2003.

[10] L. U. Choi and R. D. Murch, "Transmit-preprocessing techniques with simplified receivers for the downlink of MISO TDD-CDMA systems," IEEE Trans. on Vehicular Technology, vol. 53, no. 2, pp. 285-295, March 2004.

[11] Y. Ding, T. N. Davidson, Z.-Q. Luo, and K. M. Wong, "Minimum BER block precoders for zero-forcing equalization," IEEE Trans. on Signal Processing, vol. 51, pp. 2410-2423, Sept. 2003.

[12] K. Ban, M. Katayama, T. Yamazato, and A. Ogawa, "Transmitter precoding with multiple transmit/receive antennas for high data rate communication in bandwidth-limited channels," in Proc. of the Vehicular Technology Conf. (VTC 1999 - Fall), Sept. 1999.

[13] J. Yang and S. Roy, "Joint transmitter receiver optimization for multi input multi output systems with decision feedback," IEEE Trans. on Information Theory, vol. 40, pp. 1334-1347, Sept. 1994.

[14] A. Scaglione, P. Stoica, S. Barbarossa, G. B. Giannakis, and H. S. Sampath, "Optimal designs for space time linear precoders and decoders," IEEE Trans. on Signal Processing, vol. 50, no. 5, pp. 1051-1064, May 2002.

[15] D. P. Palomar, J. M. Cioffi, and M. A. Lagunas, "Joint Tx-Rx beamforming design for multicarrier MIMO channels: A unified framework for convex optimization," IEEE Trans. on Signal Processing, vol. 51, no. 9, Sept. 2003. 
[16] D. P. Palomar, M. A. Lagunas, and J. M. Cioffi, "Optimum linear joint transmit-receive processing for MIMO channels with QoS constraints," IEEE Trans. on Signal Processing, vol. 52, no. 5, pp. 1179-1197, May 2004.

[17] Z. Q. Luo, T.N. Davidson, G.B. Giannakis, and K.M. Wong, "Transceiver optimization for block-based multiple access through ISI channels," IEEE Trans. on Signal Processing, vol. 52, no. 4, pp. 1037-1052, April 2004.

[18] S. Boyd and L. Vandenberghe, Introduction to Convex Optimization with Engineering Applications, Stanford, 2003.

[19] R. Esmailzadeh, E. Sourour, and M. Nakagawa, "Pre-rake diversity combining in time division duplex CDMA mobile communications," IEEE Trans. on Vehicular Technology, vol. 48, no. 3, pp. 795-801, May 1999.

[20] L. U. Choi and R. D. Murch, "Transmit MMSE pre-RAKE pre-processing with simplified receivers for the downlink of MISO TDD-CDMA systems," in Proc. of Global Telecommunications Conference, 2002 (GLOBECOM-2002), 2002, pp. 17-21.

[21] K. N. Modi, K. Reynolds, and X. Wang, "Linear precoding for multi antenna CDMA onver multipath ISI channels," in Proc. of conference on Information Sciences and Systems, Princeton, (CISS-2004), March 2004.

[22] M. Brandt Pearce and A. Dharap, "Trasmitter based multiuser interference rejection for the downlink of a wireless CDMA system in a multiplath environment," IEEE Jour. on selected areas in Communications, vol. 18, pp. 407-417, March 2000.

[23] M. Meurer, P. W. Baier, T. Weber, Y. Lu, and A. Papathanassiou, "Joint transmission: advantageous downlink concept for CDMA mobile radio systems using time division duplexing," Electronics Letters, vol. 36, no. 10, pp. 900-901, May 2000.

[24] S. Georgoulis and D. Cruickshank, "Pre-equalization, transmitter precoding, and joint transmission techniques for time division duplex CDMA," in Proc. of IEE 3G Mob. Communications Technology, London,, March 2001, pp. 257-261.

[25] A. N. Barreto and G. Fettweis, "Joint signal precoding in the downlink of spread spectrum systems," IEEE Trans. on Wireless Communications, vol. 2, no. 3, pp. 511-518, May 2003. 
[26] S. L. Georgoulis and D. G. M. Cruickshank, "Transmitter-based inverse filters for reducing MAI and ISI in CDMA-TDD downlink," IEEE Trans. on Wireless Communications, vol. 3, no. 2, pp. 353-358, March 2004.

[27] J. Choi and S. Perreau, "MMSE multiuser downlink multiple antenna transmission for CDMA channels," IEEE Trans. on Signal Processing, vol. 52, no. 6, pp. 1564-1573, June 2004.

[28] S. Wang and J. Caffery, "Linear multiuser precoding for synchronous CDMA," in Proc. of IEEE Wireless Communications and Networking Conference (WCNC2003), 2003.

[29] L. U. Choi and R. D. Murch, "A transmit preprocessing technique for multiuser MIMO systems using a decomposition approach," IEEE Trans. on Wireless Communications, vol. 3, no. 1, pp. 20-24, Jan. 2004 .

[30] L. Collin, O. Berder, P. Rostaing, and G. Burel, "Optimal minimum distance based precoder for MIMO spatial multiplexing systems," IEEE Trans. on Signal Processing, vol. 52, no. 3, pp. 617-627, March 2004.

[31] R. Fischer, C. Windpassinger, A. Lampe, and J. Huber, "Space time transmission using TomlinsonHarashima precoding," in Proc. of 4th ITG Conf. on Source and Channel Coding, Jan. 2002.

[32] M. Schubert and H. Boche, “Joint 'dirty paper' pre-coding and downlink beamforming," in Proc. of IEEE International Symposium on Spread Spectrum Techniques and Applications (ISSSTA2002), Sept. 2002.

[33] C. Peel, B. Hochwald, and L. Swindlehurst, "A vector-perturbation technique for near-capacity multiantenna multi-user communication," in Proc. of the 41st Allerton Conference on Communication, Control, and Computing,, Oct. 2003.

[34] E. S. Hons, A. K. Khandani, and W. Tong, "An optimized transmitter precoding scheme for synchronous DS-CDMA,” in Proc. of IEEE Int. Communications Conf., April 2002.

[35] H. Sampath, P. Stoica, and A. Paulraj, "Generalized linear precoder and decoder design for MIMO channels using the weighted MMSE criterion," IEEE Trans. on Communications, vol. 49, no. 12, pp. 2198-2206, Dec. 2001.

[36] S. Serbetli and A. Yener, "Transceiver optimization for multiuser MIMO systems," IEEE Trans. on Signal Processing, vol. 52, no. 8, pp. 214-226, August 2004. 
[37] P. Viswanath and D. N. C. Tse, "Optimal sequences, power control, and user capacity of synchronous CDMA systems with linear MMSE multiusers receivers," IEEE Trans. on Information Theory, vol. 45, pp. 1968-1983, Sept. 1999.

[38] S. Ulukus and R. Yates, "Iterative construction of optimum signature sequence sets in synchronous cdma systems," IEEE Transactions on Information Theory, vol. 47, no. 5, July 2001.

[39] T. Guess, "Optimal sequences for CDMA with decision feedback receivers," IEEE Trans. on Information Theory, vol. 49, pp. 886-900, April 2003.

[40] J. H. Chang, L. Tassiulas, and F. Rashid Farrokhi, "Joint transmitter receiver diversity for efficient space division multiple access," IEEE Trans. on Wireless Communications, vol. 1, pp. 16-27, Jan. 2002.

[41] E. Visotsky and U. Madhow, "Optimum beamforming using transmit antenna arrays," in Proc. of IEEE Vehicular Technology Conf., May 1999, vol. 1, pp. 851-856.

[42] M. Bengtsson and B. Ottersten, "Optimal downlink beamforming using semidefinite optimization," in Proc. of 37th Annual Allerton, Sept. 1999.

[43] H. Boche and M. Schubert, "A general duality theory for uplink and downlink beamforming," in Proc. of IEEE Vehicular Technology Conf. (VTC-2002 Fall), Sept. 2002, pp. 87-91.

[44] P. Viswanath and D. N. C. Tse, "Sum capacity of the vector gaussian broadcast channel and uplink downlink duality," IEEE Trans. on Information Theory, vol. 49, pp. 1912-1921, Aug. 2003.

[45] D. N. C. Tse and P. Viswanath, "Downlink uplink duality and effective bandwidths," in Proc. of IEEE International Symposium on Information Theory (ISIT-2002), June 2002.

[46] F. Rashid Farrokhi, L. Tassiulas, and K. J. Ray Liu, "Joint optimal power control and beamforming in wireless networks using antenna arrays," IEEE Trans. on Communications, vol. 46, pp. 1313-1324, Oct. 1998.

[47] Tai-Lai Tung and Kung Yao, "Optimal downlink power-control design methodology for a mobile radio DS-CMDA system," in Proc. of IEEE workshop on Signal Processing Systems (SIPS-2002), 2002, pp. $165-170$. 
[48] M. Schubert, D. Karadoulamas, H. Boche, and G. Lebmann, "Joint downlink beamforming and power control for 3G WCDMA," in Proc. of IEEE Vehicular Technology Conf. (VTC-2003 Spring), April 2003, vol. 1, pp. 331-335.

[49] M. Schubert and H. Boche, "Solution of the multiuser downlink beamforming problem with individual SINR constraints," IEEE Trans. on Vehicular Technology, vol. 53, no. 1, pp. 18-28, Jan 2004.

[50] S. Shi and M. Schubert, "Precoding and power loading for multi antenna broadcast channels," in Proc. of conference on Information Sciences and Systems, Princeton, (CISS-2004), March 2004.

[51] J. F. Sturm, "Using SEDUMI 1.02, a Matlab toolbox for optimizations over symmetric cones," Optimization Meth. and Soft., vol. 11-12, 1999.

[52] M. S. Lobo, L. Vandenberghe, S. Boyd, and H. Lebret, "Applications of second order cone programming," Linear Algebra and App., 1998.

[53] L. Vandenberghe and S. Boyd, "Semidefinite programming," Siam Review, vol. 38(1), pp. 49-95, March 1996.

[54] S. Boyd and L. E. Ghaoui, "Method of centers for minimizing generalized eigenvalues," Linear Algebra and App., April 1992.

[55] C. D. Meyer, Matrix analysis and applied linear algebra, Siam, 2000.

[56] R. Yates, "A framework for uplink power control in cellular radio systems," IEEE Journal on Selected Areas in Communications, vol. 13, no. 7, Sept. 1995.

[57] Y. C. Eldar, "Minimax MSE-ratio estimation with bounded data uncertainties," in submitted to IEEE Trans. Signal Processing, Dec. 2003.

[58] P. Gahinet, A. Nemirovski, A. J. Laud, and M. Chilali, LMI Control Toolbox, Matlab, the Mathworks.

[59] A. Ben-Tal and A. Nemirovski, "Robust optimization methodology and applications," Math Programming, vol. 92, pp. 453-480, 2002. 